\title{
Hybrid LES / RANS Simulation of Transverse Sonic Injection into a Mach 2 Flow
}

\author{
John A. Boles* and Jack R. Edwards ${ }^{\dagger}$ \\ North Carolina State University, Raleigh, NC \\ Robert A. Baurle ${ }^{\ddagger}$ \\ NASA Langley Research Center, Hampton, VA
}

\begin{abstract}
A computational study of transverse sonic injection of air and helium into a Mach 1.98 cross-flow is presented. A hybrid large-eddy simulation / Reynolds-averaged Navier-Stokes (LES/RANS) turbulence model is used, with the two-equation Menter-BSL closure for the RANS part of the flow and a Smagorinsky-type model for the LES part of the flow. A timedependent blending function, dependent on modeled turbulence variables, is used to shift the closure from RANS to LES. Turbulent structures are initiated and sustained through the use of a recycling / rescaling technique. Two higher-order discretizations, the Piecewise Parabolic Method (PPM) of Colella and Woodward, and the SONIC-A ENO scheme of Suresh and Huyhn are used in the study. The results using the hybrid model show reasonably good agreement with time-averaged Mie scattering data and with experimental surface pressure distributions, even though the penetration of the jet into the cross-flow is slightly over-predicted. The LES/RANS results are used to examine the validity of commonly-used assumptions of constant Schmidt and Prandtl numbers in the intense mixing zone downstream of the injection location.
\end{abstract}

\section{Introduction}

U NDERSTANDING the flow physics involved with sonic injection into a supersonic cross-flow plays a vital role in the analysis and design of hypersonic engines. In a hypersonic combustor, it is critical that the injected fuel be sufficiently mixed with the oxidizer so that combustion may occur. Achieving adequate mixing within a hypersonic engine is non-trivial, since the flow residence time in these devices is often comparable to the chemical time scales. As a result, studying the impacts of using different fuels and injection schema is vitally important to further advance the design of combustors for air-breathing hypersonic flight. Unfortunately, experimental studies of these complex flows are costly to perform and reliable measurements are often difficult to obtain, forcing an increased reliance on computational studies to augment the databases of sonic injection into supersonic flowfields.

Early experimental studies [1-6] used optical techniques to analyze wind tunnel experiments. Andrepoulos [6] authored an extensive review of the experimental work in injection studies up to 1984. Figure 1 shows the qualitative view of the flowfield features that result from transverse injection into a supersonic cross-flow. Optical methods allowed researchers to discern the major flow phenomena shown in this figure such as the prominent bow shock, Mach disk in the interior of the injection plume, counter-rotating vortices in the injection plume, and the wake and vortex regions beneath the injection plume. More recent studies [7-12] utilized laser-tracking technology in addition to optical techniques to glean more information from wind tunnel tests. Even with newer technology, quantitative results are often limited.

Computational studies can also offer insight into the mechanics of these flows. Tam, et al. [13] used the MenterBSL and Menter-SST [14] RANS models as well as the k- $\omega$ model of Wilcox [15] to simulate the sonic injection experiments of Gruber, et al [9-12]. It was shown that the BSL model and the Wilcox model provided the best agreement with the surface pressure measurements of Gruber. All of the models tested, however, over-predicted the penetration of the injectant by up to $25 \%$.

\footnotetext{
${ }^{*}$ Research Assistant, Department of Mechanical and Aerospace Engineering, Student Member, AIAA.

${ }^{\dagger}$ Professor, Department of Mechanical and Aerospace Engineering, Associate Fellow, AIAA.

‡ Aerospace Engineer, Hypersonic Air Breathing Propulsion Branch, Senior Member, AIAA
} 
Palekar, et al. [16] attempted to simulate this same experiment using the commercial CFD software GASP with Wilcox's k- $\omega$ model with better success than Tam, et al. The injectant penetration height was lower and correlated fairly well with the measurements except in the spanwise direction, where significant deviations from the measurements were noted. This improvement in performance may be due to the use of finer grids in this study, as the turbulence models used were similar to those of Tam, et al. Palekar, et al. performed a grid refinement study with three different grids: a 4.7 million-cell mesh, a 610,000 cell mesh, and an 81,000 cell mesh. This study revealed that the coarse and medium grids were not capable of adequately resolving all of the relevant flow features.

Maddalena [17] simulated an aeroramp injection scheme as well as the transverse injection of sonic helium into a Mach 4 airstream. Again the Wilcox k- $\omega$ model was used in conjunction with the GASP solver. This study showed little advantage to using the aeroramp injector, and the total pressure loss was greater than that obtained for the normal injection configuration. The computational results did not correlate well with this experimental data set. The author suggested that the lack of upstream turbulence in the model may be to blame for the lack of agreement.

Manna [18] simulated a two-stage transverse injector downstream of a backward-facing step. The cross-flow was Mach 2 air with sonic air injected in two transverse circular injectors. The commercial CFD solver CFX-TASCFlow was used along with the k- $\varepsilon$ model. The study showed good correlation with experimental data away from the injectors but differences were noted in the injector near-field. These discrepancies were attributed to the assumption of uniform flow at the injector and to the inadequacy of the turbulence model.

Steady-state RANS simulations were utilized in all of the above studies because the Reynolds numbers were high enough to render a wall-resolved LES or direct numerical simulation impractical. Peterson and co-workers [19] utilized a detached-eddy simulation approach on an unstructured mesh to simulate the sonic-injection experiments conducted at Virginia Polytechnic University. They were able to obtain acceptable results by forcing the inflow boundary layer using a population of hairpin-type vortices generated through a separate calculation. In the present work, a hybrid LES/RANS method [20-23] is used to simulate the experiments of Gruber and co-workers [9-12], which involve the injection of sonic air or helium into a Mach 1.98 air cross-flow. This study is part of a continuing effort to develop hybrid LES/RANS strategies that are more suited for computing strongly-interacting, threedimensional wall-bounded flows encountered in high-speed engine concepts. The viewpoint adopted in this work is that the RANS component functions as a type of near-wall turbulence closure, with the shift from RANS to LES governed by a flow-dependent blending function that is designed to transition (in a time-averaged sense) from RANS to LES near the edge of the logarithmic region of the boundary layer. Recycling / rescaling techniques are used to initiate and sustain large-eddy structures at inflow boundaries, and in this study, higher-order extensions based on the Piecewise Parabolic Method [24] and the SONIC-A essentially non-oscillatory (ENO) scheme [25] are compared for air injection into the cross-flow. The remainder of this paper describes the turbulence closure models used, the numerical methods, the initialization procedure, and the results of the investigation.

\section{Numerical Model}

\section{A. Governing Equations and Turbulence Model}

The Favre-filtered Navier-Stokes equations, expanded to include separate transport equations for individual species, are solved in a finite volume form in this study. The interpretation of the averaging process in a general sense leads to a similarity between the Favre-filtered form used in large-eddy simulation and the Favre-averaged form used in unsteady Reynolds-averaged Navier-Stokes modeling. As such, the same set of equations can be solved in a time-dependent manner, and the shift between an LES interpretation and an unsteady RANS interpretation depends on the form of the model for the unresolved stress, diffusion, and conduction terms. In this study, a simple approach based on Boussinesq / gradient diffusion assumptions is used to facilitate this shift. Constant values for the turbulent Schmidt and Prandtl numbers (0.9 and 0.9) are assumed for species diffusion and heat conduction (these choices primarily affect the RANS component of the closure). With these assumptions, the closure problem reduces to specifying the eddy viscosity.

The hybrid LES/RANS model used in this investigation [23] is based on Menter's k- $\omega$ / k- $\varepsilon$ model and only involves modifications to the eddy viscosity description:

$$
\mu_{t}=\rho v_{t}=\rho\left(\Gamma \frac{k}{\omega}+(1-\Gamma) v_{t, s g s}\right),
$$

As the blending function $\Gamma$ approaches one, the closure approaches its RANS description, and as it approaches zero, a subgrid eddy viscosity is obtained. This work uses a Smagorinsky model for the subgrid viscosity, defined as: 


$$
\begin{aligned}
& v_{t, s g s}=C_{s} \Delta^{2} S, C_{s}=0.01, \quad \Delta=\left(\Delta_{x} \Delta_{y} \Delta_{z}\right)^{1 / 3} \\
& S=\left[\frac{\partial \tilde{u}_{i}}{\partial x_{j}} \frac{\partial \tilde{u}_{j}}{\partial x_{i}}+\frac{\partial \tilde{u}_{i}}{\partial x_{j}} \frac{\partial \tilde{u}_{i}}{\partial x_{j}}-\frac{2}{3}\left(\frac{\partial \tilde{u}_{i}}{\partial x_{i}}\right)^{2}\right]^{1 / 2}
\end{aligned}
$$

The blending function $\Gamma$ is based on the ratio of the wall distance $d$ to a modeled form of the Taylor micro-scale:

$$
\Gamma=\frac{1}{2}\left(1-\tanh \left[5\left(\frac{\kappa}{\sqrt{C_{\mu}}} \eta^{2}-1\right)-\phi\right]\right), \quad \eta=\frac{d}{\alpha_{1} \lambda}
$$

where the Taylor micro-scale is defined as $\lambda=\sqrt{\nu / C_{\mu} \omega}$, and $\phi$ is set to $\tanh ^{-1}(0.98)$ in order to fix the balancing position (i.e. where $\frac{\kappa}{\sqrt{C_{\mu}}} \eta^{2}=1$ ) to $\Gamma=0.99$. The constant $\alpha_{1}$ is chosen to force the average LES to RANS transition position ( $\Gamma=0.99$ ) for equilibrium boundary layers to occur at the point where the wake law starts to deviate from the log law. To determine $\alpha_{1}$ for a particular inflow boundary layer, the procedure taken from Ref. [23] is used. First, a prediction of the equilibrium boundary layer is obtained (given free-stream properties, a specified wall thermal condition, and a value for the boundary layer thickness) from Coles' Law of the Wall / Wake along with the Van Driest transformation:

$$
\frac{u_{v d}}{u_{\tau}}=\frac{1}{\kappa} \ln \left(d_{w}^{+}\right)+C+2 \frac{\Pi}{\kappa} \sin ^{2}\left(\frac{\pi}{2} \frac{d}{\delta}\right), d_{w}^{+}=\frac{u_{\tau} d}{v_{w}}
$$

An initial estimate for the outer extent of the log layer is defined by finding the value of $d_{w}^{+}$such that $\left(\frac{1}{\kappa} \ln \left(d_{w}^{+}\right)+C\right) /\left(\frac{u_{v d}}{u_{\tau}}\right)=0.98$. The value of $d^{+}=u_{\tau} d / v$ that corresponds to this value of $d_{w}^{+}$is then found through the use of Walz's formula [26] for the static temperature distribution within the boundary layer. The model constant is then obtained by the equivalence $d^{+}=\alpha_{1}^{2}$, which arises from the use of inner-layer scaling arguments for $k$ and $\omega$. For the inflow boundary layer used in this investigation ( $\delta=6.35 \mathrm{~mm}$, Mach number of 1.98, adiabatic wall), a value of $\alpha_{1}=37.56$ was calculated. A recycling / rescaling procedure [21,22], applied to the fluctuating fields, was used to sustain grid-resolved turbulent structures in the outer part of the incoming boundary layer.

\section{B. Numerical Methods}

A planar relaxation sub-iteration procedure coupled with a Crank-Nicholson time discretization was used to advance the governing equations at second-order temporal accuracy. Details on the calculation of the unsteady residual and flux Jacobians can be found in Ref. 27. Inviscid fluxes were calculated using Edwards' LDFSS upwind scheme [27]. These fluxes were extended to second-order accuracy using either the SONIC-A ENO technique [25], or the Piecewise Parabolic Method (PPM) [24]. The reconstruction procedures were applied to the primitive variable vector $V=\left[\rho_{s}, u, v, w, T, k, \omega\right]$, where $\rho_{s}$ is the species density. The code used to perform the simulations has been parallelized using three-dimensional, spatial domain decomposition with MPI communication.

\section{Initial Condition and Time-advancement Details}

To provide an initial condition for the incoming boundary layer, a two-dimensional simulation was performed for the facility nozzle [28]. The placement of the injector port was just downstream of the start of the test section, and the boundary layer thickness in the vicinity of the injector was reported to be approximately the same as the diameter of the injector $(6.35 \mathrm{~mm})$. Accordingly, the streamwise location of the injector center-line was placed at the position where the boundary layer thickness for the converged RANS solution was $6.35 \mathrm{~mm}$. The inflow plane for the calculation was placed at approximately 12 injector diameters upstream of this location. A three-dimensional mesh with clustering to the injector location and to the walls was then generated. This mesh extends $+/-12$ injector diameters in the streamwise (x) direction, +/- 6 injector diameters in the spanwise (z) direction, and 16 injector 
diameters in the transverse (y) direction. A metric of approximately 15 cells / incoming boundary layer thickness was used in the isotropic ( $\mathrm{x}$ and $\mathrm{z}$ ) directions and in the wall normal (y) region traversed by the jet plume, leading to about 11.8 million cells. The mesh was clustered to the plate surface and to the injector location, thus the resolution near the injector is finer than the 15 cells / boundary layer thickness metric used for the boundary layer of the approach flow. About 625 cells were located over the $\mathrm{x}-\mathrm{z}$ region occupied by the circular injector port. The exit conditions for the injectant were obtained by computing the axisymmetric flow within the injector nozzle. This solution was then interpolated to the ghost cells associated with the injector port, but no attempt was made to match the mesh topologies, and time-dependent forcing of the jet fluid was not attempted. Periodic boundary conditions were applied in the $\mathrm{z}$ direction, and extrapolation conditions were used at the upper extent of the domain in the $\mathrm{y}$ direction and at the downstream end in the $\mathrm{x}$ direction. The simulations therefore correspond to injection into an unbounded domain in the $y$ and $\mathrm{z}$ planes, and possible blockage effects caused by boundary layer growth on the side and upper walls of the test section are not accounted for. These concessions may affect the predictions of jet penetration, as discussed later. A Menter BSL RANS solution with injection was then obtained and used as the initial condition for the hybrid simulations. The time-dependent calculations were simulated for approximately 5 flow through times in order to remove start-up transients, then statistics were collected for approximately 7 milliseconds of physical time ( 24 flow through times, based on the free-stream conditions and domain length) at a time step of 0.25 microseconds.

\section{Results and Discussion}

\section{A. Inflow Boundary Layer}

The velocity boundary layer profiles as calculated by the hybrid LES / RANS formulations are shown in Figure 2. The results in Figure 2a are normalized by the free-stream velocity $(519 \mathrm{~m} / \mathrm{s})$ and by the target boundary layer thickness $(0.00635 \mathrm{~m})$. This figure shows that the PPM approach (as compared with the SONIC-A method at the same location) predicts a slightly thicker boundary layer, yields higher velocities near the wall, and provides a larger resolved Reynolds shear stress. The SONIC-A results are in closer agreement with the RANS solution. Also shown are results from a reference experiment at zero pressure gradient [29]. This comparison indicates that the computed boundary layers have not yet returned to equilibrium. When the profiles are plotted using wall coordinates (Figure 2b), it is seen that the PPM predictions adhere to the logarithmic law more closely than those obtained using the SONIC-A scheme or the Menter RANS model. The Van Driest-transformed velocity values for the SONIC-A scheme are higher than those obtained from the PPM solution, but the non-transformed velocity values are lower. This indicates that the friction velocity predicted by the SONIC-A scheme is lower than the value predicted by the PPM approach. More recent work [30] has shown that the enhanced acceleration toward the surface and the increased boundary layer growth rate observed in the PPM calculations are the result of determining the pressure field at cell interfaces from the reconstructed species density and temperature information instead of reconstructing the pressure field directly.

\section{B. Case 1 - Sonic Air injected into Mach 1.98 Air}

Figure 3 compares the computed center-line pressure distribution with the experimental data of Gruber, et al. Both hybrid simulations captured the pressure drop upstream of the injector, while the RANS model did not - the PPM results reflected the experimental data particularly well in this regard. Moreover, while every model predicted a delayed recovery of the pressure downstream of the injector relative to the experimental measurements, the hybrid models predicted the correct pressure peak. The over-prediction of the center-line surface pressure in the region just downstream of the injector is also evident in the transverse pressure profiles in Figure 4. The experimental profiles at locations upstream of the injector are well-predicted by each model, indicating that the upstream extent of the separation region induced by the bow shock is captured correctly. Downstream of the first three stations, all of the models under-predict the off-center pressure profiles, while the RANS model over-predicts the pressure peak at the center of the last three stations. The slight asymmetry in the LES/RANS profiles suggests a lack of complete statistical convergence.

Figure 5 shows snapshots of inlet air species density at the $x-y$ center-plane, and at a cross-flow station located at $\mathrm{x} / \mathrm{D}=$ 5.9. An intense near-field mixing process, characterized by a dominant pair of counter-rotating, streamwiseoriented vortices and shear-layer Kelvin-Helmholtz structures, is illustrated in the density contours. Time-averaged inlet air density contours are compared with Mie-scattering data provided by Gruber, et al. [9] in Figure 6. Good agreement is generally indicated. Figure 7a shows the time-averaged mass fraction at the $x-y$ center-plane, with the $x$ 
and y coordinates normalized by the product of the momentum flux ratio $(\mathrm{J}=2.90)$ and the injector diameter. The results are compared with a penetration curve [11] determined experimentally from the averaged Mie-scattering data by mapping the $90 \%$ intensity contour, determined relative to an arbitrary value behind the bow shock. The penetration curve (which is valid up to $\sim(\mathrm{x}+\mathrm{r}) / \mathrm{J} * \mathrm{D})=1.5$ ) bisects the outer edge of the jet shear layer. Figures $7 \mathrm{~b}$ and 7c compare the inlet air density standard deviation with the experimental intensity standard deviation (normalized to lie between zero and one). The density standard deviations were determined over a sample space of only 50 frames, which is comparable to the experimental sample space of 100 frames. The results are thus not as smooth as those shown in Figure 6 and Figure 7a, which were obtained by averaging over 24000 frames. Figures $7 \mathrm{~b}$ and $7 \mathrm{c}$ indicate close agreement with the structural features shown in the experiment, but it is clear that the jet penetration is slightly over-predicted.

Comparisons of predicted inlet air density with experimental Mie-scattering images at $x / D=0, x / D=4, x / D=8$, and $\mathrm{x} / \mathrm{D}=10$ are shown in Figure 8 . The results from the hybrid models show relatively good agreement with the positions of the jet plume at each station. The bow shock at the first station is also distinct and consistent with the experimental data. The wake vortices beneath the jet plume are more sharply defined for the PPM scheme than for the SONIC-A scheme. The RANS solution shows very different plume shapes that display more distinct vortical features as well as sharp transitions between the jet and inlet-air streams. Considering the differences in the numbers of frames used in the averaging ( 24000 in the calculations versus 50 or 100 in the experiments), and in the use of arbitrary intensities in normalizing the Mie-scattering images, it is difficult to compare the darkening / lightening of the contours in any quantitative sense. Visually, though, it appears that the plumes in the experiment are more elongated in the transverse direction and tend to narrow in the spanwise (lateral) direction as one proceeds downstream. In contrast, the computational plumes appear to expand in a more uniform manner in the $\mathrm{y}$ and $\mathrm{z}$ directions. This may be an indication of the effects of flow confinement in the experiment.

For a more precise comparison between the SONIC-A and PPM schemes, the $90 \%$ cross-flow mass fraction contour is shown for each in Figure 9. The contours are similar at the first two stations. At the third and fourth stations, the SONIC-A scheme predicts a wider plume at the bottom, and the entire plume is also slightly lower than that obtained using the PPM scheme. Also, the PPM results show a more pronounced double vortex structure in the plume. This is likely due to the fact that the transition from injectant to cross-flow air is sharper when the PPM scheme is used; thus the $10 \%$ injectant contour is closer to the vortex centers than for the more diffuse SONIC-A solution.

\section{Case 2 - Sonic Helium injected in Mach 1.98 Air}

A simulation of sonic injection of helium into the Mach 1.98 cross-flow using the PPM scheme with the hybrid LES / RANS model has also been performed. Figure 10 shows instantaneous snapshots of inlet air density for this case. In comparison with Figure 5, one can note darker contours within the plume, indicating less large-scale entrainment of the jet fluid into the air stream. The eddy sizes appear to be slightly smaller for the helium injection than was observed for the air injection case. Time-dependent animations examined for both air and helium injection showed that the dynamics of the shock system influence the initial growth of Kelvin-Helmholtz structures in the outer part of the jet shear layer. Time-averaged mass fraction distributions and standard deviation comparisons are shown in Figure 11. The experimental penetration curve lies closer to the outer edge of the jet than in the air injection case (Figure 11a), and visual comparisons of Figures 11b and 11c indicate good agreement with the structural features of the jet. The jet penetration rate appears to be better predicted for the helium injection case than for the air injection case (Figure 6). Figure 12, however, shows several discrepancies in the cross-plane structure of the plume. The simulation predicts a wider plume at $\mathrm{x} / \mathrm{D}=8$ and $\mathrm{x} / \mathrm{D}=10$, with the bottom edges of the counterrotating vortex pairs located further from the bottom wall than indicated in the Mie-scattering data. In contrast, the counter-rotating vortex pair is less well-defined in the predictions at $x / D=8$, relative to the experimental image.

\section{Penetration comparisons}

Transverse and spanwise injectant penetration values for both cases are compared with the data of Gruber, et al. $[9,11]$ in Figure 13. Computational transverse penetration values at a particular $\mathrm{x} / \mathrm{D}$ station are found by determining the location of the $90 \%$ injectant mass fraction contour (Figure 9) at its outermost point. The transverse penetration profiles (Figure 13a) show that the locations of the tops of the plumes are predicted fairly well for the air case at $\mathrm{x} / \mathrm{D}$ $=0$ and $\mathrm{x} / \mathrm{D}=4$, even though the visual comparisons of Figure 7 suggest more penetration in the calculations. At $\mathrm{x} / \mathrm{D}=8$ and $\mathrm{x} / \mathrm{D}=10$, the Gruber, et al. results indicate that the top of the plume moves closer to the wall. This is not reflected in any of the simulation results. The experimental trends of increasing transverse penetration with 
increasing distance from the injector are captured in the helium-injection results, but the values are under-predicted. This is in contrast with the visual comparison of the intensity standard deviation images shown in Figure 11, which indicates close agreement. Spanwise penetration is a measurement of the maximum span of the plume (again determined by the $90 \%$ injectant mass fraction contour), normalized by the injector nozzle diameter. For the air injection case, the spanwise penetration (Figure 13b) is similar to the experimental data for the first two stations, but the downward trend of the spanwise penetration indicated in the experimental data at the last two stations is not seen in the calculations. This corresponds to the narrowing of the plume indicated in the Mie-scattering images (Figure 8), which is not captured in the calculations. Palekar, et al. [16] saw a similar discrepancy in their spanwise penetration results. The experimental spanwise penetration data for the helium plume does not show a substantial decrease at the last two stations, even though the images do indicate some elongation of the plume in the transverse direction. The calculation matches the lateral expansion rate of the helium plume, but again underpredicts the measured values.

\section{E. Calculation of Turbulent Prandtl and Schmidt Numbers}

A significant source of uncertainty in RANS calculations of high-speed engine flowfields is the specification of the turbulent Prandtl and Schmidt numbers. These scale the turbulent heat conduction and diffusion terms, which are typically modeled using gradient diffusion assumptions. Several studies [31, for example] have shown an extreme sensitivity of RANS calculations to the choices of the turbulent Prandtl and Schmidt numbers. While models exist to calculate the variation of these quantities as part of a RANS solution [32,33], their development has been hampered by the lack of detailed experimental data for the turbulent scalar fluxes. One of the advantages of a large-eddy simulation approach is that larger-scale transport mechanisms are directly captured, and given a large-eddy simulation (or hybrid LES/RANS) database, it is possible to extract Favre-averaged distributions of fluxes of mass, momentum, and energy due to turbulent fluctuations and to compare them with Boussinesq / gradient-diffusion closures commonly used in RANS modeling. A flux of a variable $\phi^{\prime}=\left[Y^{\prime}, u_{i}^{\prime}, T^{\prime}\right]$ may be expressed as

$$
\overline{\rho u_{j}^{\prime} \phi^{\prime}}=\overline{\rho u_{j} \phi}-\frac{\overline{\rho u_{j}} \overline{\rho \phi}}{\bar{\rho}},
$$

where the over-bars indicate time averages. For turbulent mass transport, the value above may be correlated with a gradient-diffusion parameterization:

$$
\overline{\rho u_{j}^{\prime} Y^{\prime}}=-\bar{\rho} v_{t, \text { mass }, j} \frac{\partial \tilde{Y}}{\partial x_{j}},
$$

where a Favre-averaged variable $\tilde{\phi}\left(\phi=\left[Y, u_{i}, T\right]\right)$ is defined as $\tilde{\phi}=\frac{\overline{\rho \phi}}{\bar{\rho}}$. In general, separate 'eddy viscosities' $\left(v_{t, \text { mass }, j}\right)$ could be determined for each component of the gradient vector. The validity of the gradient-diffusion assumption requires that $\overline{\rho u_{j}^{\prime} Y^{\prime}}$ be well-correlated with the gradient in mass fraction. We have verified that this is case for the mixing region downstream of injection, particularly for the $\mathrm{y}$ and $\mathrm{z}$ directions. As RANS models will typically use an isotropic eddy viscosity, Eq. (6) can be rewritten as $\overline{\rho u_{j}^{\prime} Y^{\prime}}=-\bar{\rho} v_{t, m a s s} \frac{\partial \tilde{Y}}{\partial x_{j}}$, and an effective isotropic eddy viscosity associated with mass transport can be determined by taking an L2 norm of the turbulent diffusion velocity vector, i.e.:

$$
v_{t, \text { mass }} \approx-\frac{\left\langle\overline{\rho u_{i}^{\prime} Y^{\prime}} \frac{\partial \tilde{Y}}{\partial x_{i}}\right\rangle}{\left\langle\bar{\rho} \frac{\partial \tilde{Y}}{\partial x_{i}} \frac{\partial \tilde{Y}}{\partial x_{i}}\right\rangle}
$$

Here, the outer brackets denote a spatial filtering operation used to reduce statistical fluctuations. Similarly, one can define isotropic eddy viscosities associated with momentum transport and energy transport as follows:

$v_{t, \text { mom }} \approx-\frac{\left.\left\langle\overline{\left(\rho u_{i}^{\prime} u_{j}^{\prime}\right.}-\frac{2}{3} \delta_{i j} \overline{\rho k}\right) S_{i j}\left(\tilde{u}_{i}\right)\right\rangle}{\left\langle\bar{\rho} S_{i j}\left(\tilde{u}_{i}\right) S_{i j}\left(\tilde{u}_{i}\right)\right\rangle}, S_{i j}\left(\tilde{u}_{i}\right)=\frac{\partial \tilde{u}_{i}}{\partial \tilde{u}_{j}}+\frac{\partial \tilde{u}_{j}}{\partial \tilde{u}_{i}}-\frac{2}{3} \frac{\partial \tilde{u}_{k}}{\partial \tilde{u}_{k}}, \overline{\rho k}=\frac{1}{2}\left(\overline{\rho u_{k} u_{k}}-\frac{\overline{\rho u_{k}} \overline{\rho u_{k}}}{\bar{\rho}}\right)$ 


$$
v_{t, \text { energy }} \approx-\frac{\left\langle\overline{\rho u_{i}^{\prime} T^{\prime}} \frac{\partial \tilde{T}}{\partial x_{i}}\right\rangle}{\left\langle\bar{\rho} \frac{\partial \tilde{T}}{\partial x_{i}} \frac{\partial \tilde{T}}{\partial x_{i}}\right\rangle}
$$

Turbulent Schmidt and Prandtl numbers may then be calculated by taking ratios of these isotropic eddy viscosities:

$$
\frac{1}{S c_{t}}=\frac{v_{t, \text { mass }}}{v_{t, \text { mom }}} \text { and } \quad \frac{1}{\mathrm{Pr}_{t}}=\frac{v_{t, \text { energy }}}{v_{t, \text { mom }}}
$$

Figure 14a-c shows predictions of the isotropic eddy viscosities associated with turbulent momentum, mass, and energy transport for the air injection case at $\mathrm{x} / \mathrm{D}=10.9$. The calculation extent is restricted to the area bounded by the $95 \%$ inlet air mass fraction contour. There are several similarities between the momentum and energy eddyviscosity distributions, but the mass eddy-viscosity distribution shows large values near the outer region of the plume that do not appear to be highly correlated with momentum transport in this region. Scatter plots of mass eddy viscosity and energy eddy viscosity versus momentum eddy viscosity are shown in Figures 15a and 15b. A constant Schmidt or Prandtl number parameterization would result in the collapse of the data band around a line of constant slope (several such lines are indicated in the figure). The shape of the scatter-plots indicates that the average Schmidt number is very low - of the order of $1 / 10$ or less - at both $x / D=5.9$ and $x / D=10.9$. Significant deviations from this average are present, particularly in the outer regions of the plume. The eddy viscosity associated with turbulent heat transport correlates somewhat better with that of momentum, and a collapse in some of the data around Prandtl numbers of 0.25 to 0.5 start to become evident at the $\mathrm{x} / \mathrm{D}=10.9$ station. For this case, the average momentum eddy viscosity $\left(\mathrm{m}^{2} / \mathrm{s}\right)$ over the plume cross-section is 0.0196 at $\mathrm{x} / \mathrm{D}=0.0,0.0226$ at $\mathrm{x} / \mathrm{D}=5.9$, and 0.0426 at $\mathrm{x} / \mathrm{D}=10.9$. Normalized with respect to the incoming air kinematic viscosity of $1.34 \mathrm{e}-5 \mathrm{~m}^{2} / \mathrm{s}$, these values become 1463 at $\mathrm{x} / \mathrm{D}=0.0,1686$ at $\mathrm{x} / \mathrm{D}=5.9$, and 3179 at $\mathrm{x} / \mathrm{D}=10.9$. These values are similar to those provided by RANS turbulence models for free-shear layers at high Reynolds numbers.

Figure 16a-c indicates a larger degree of similarity among the different eddy viscosities for the helium case at $\mathrm{x} / \mathrm{D}=10.9$. The mass and momentum eddy viscosities are in closer agreement, with the energy viscosity deviating somewhat more from the others. Because of the different specific heats of the fluids, the turbulent heat flux also includes a contribution due to turbulent mixing, so that the full form of the turbulent heat flux becomes

$$
\begin{aligned}
\overline{\rho\left(\sum_{k} C_{p k} Y_{k}\right) T u_{j}}-\bar{\rho}\left(\sum_{k} C_{p k} \tilde{Y}_{k}\right) \tilde{T}_{\tilde{u}_{j}} & =\left(\sum_{k} C_{p k} \tilde{Y}_{k}\right)\left[\overline{\rho u_{j} T}-\frac{\overline{\rho u_{j}} \overline{\rho T}}{\bar{\rho}}\right]+\tilde{T} \sum_{k} C_{p k}\left[\overline{\rho u_{j} Y_{k}}-\frac{\overline{\rho u_{j}} \overline{\rho Y_{k}}}{\bar{\rho}}\right] \\
& +\tilde{u}_{j} \sum_{k} C_{p k}\left[\overline{\rho T Y}_{k}-\frac{\left.\overline{\rho T} \frac{\overline{\rho Y}}{\bar{\rho}}\right]+\sum_{k} \rho Y_{k}^{\prime} u_{j}^{\prime} T^{\prime}}{}\right.
\end{aligned}
$$

(11)

In conventional RANS techniques, the first term on the right-hand side is modeled by a Fourier's law parameterization with a constant turbulent Prandtl number:

$$
\left(\sum_{k} C_{p k} \tilde{Y}_{k}\right)\left[\overline{\rho u_{j} T}-\frac{\overline{\rho u_{j}} \overline{\rho T}}{\bar{\rho}}\right] \sim-\left(\sum_{k} C_{p k} \tilde{Y}_{k}\right) \frac{v_{t, \text { energy }}}{\operatorname{Pr}_{t}} \frac{\partial T}{\partial x_{j}}
$$

The second term on the right-hand side is normally modeled using gradient-diffusion concepts, which for binary mixing leads to

$$
\tilde{T} \sum_{k} C_{p k}\left[\overline{\rho u_{j} Y_{k}}-\frac{\overline{\rho u_{j}} \overline{\rho Y_{k}}}{\bar{\rho}}\right]=\tilde{T}\left(C_{p 2}-C_{p 1}\right)\left(\overline{\rho u_{j} Y_{2}}-\frac{\overline{\rho u_{j}} \overline{\rho Y_{2}}}{\bar{\rho}}\right) \sim-\frac{v_{t, m a s s}}{S c_{t}}\left(C_{p 2}-C_{p 1}\right) \tilde{T} \frac{\partial Y_{2}}{\partial x_{j}}
$$

(13)

The third and fourth terms are normally neglected in RANS modeling. Their relative importance could be assessed by computing the terms directly from the LES database - unfortunately, not enough data was stored to conduct this analysis at this time. Eqs. (12) and (13) indicate that, for binary mixing, the expressions in Eqs. (8) and (9) still hold as a means of estimating the isotropic eddy viscosities for the helium injection case, but the absence of the temperature-species correlation in particular may lead to errors in interpretation, relative to the case where the species have identical specific heats. The average momentum eddy viscosities $\left(\mathrm{m}^{2} / \mathrm{s}\right)$ are higher than in the air injection case: 0.0532 (normalized - 3941) at $\mathrm{x} / \mathrm{D}=0.0 ; 0.200$ (normalized -14925 ) at $\mathrm{x} / \mathrm{D}=5.9$, and 0.132 (normalized - 9892) at $\mathrm{x} / \mathrm{D}=10.9$. In a RANS context, these differences appear to be associated with the larger velocity difference between the streams and imply more turbulence production associated with stronger velocity gradients. 
The scatter plots in Figure 17a indicate that the mass and momentum eddy viscosities start to collapse toward the line associated with a Schmidt number of approximately 0.5 at the $x / D=10.9$ station. At the $x / D=5.9$ station, a bimodal behavior is indicated, with some data arranged about the $\mathrm{Sc}_{\mathrm{t}}=1.0$ line and the rest oriented about the $\mathrm{Sc}_{\mathrm{t}}=$ 0.5 line. For low molecular-weight fluids, a value of $\mathrm{Sc}_{\mathrm{t}}=0.5$ is commonly adopted in RANS calculations, and it is noteworthy that the LES/RANS database predicts a collapse around this value at the most downstream station. The helium case appears to be less dominated by large-scale entrainment effects (macro-mixing) than the air case, and the fact that mixing appears to be occurring at a smaller scale may lead to a faster equilibration of the rates of mass and momentum transport. The scatter plots relating energy and momentum eddy viscosities (Figure 17b) do not show a clear collapse about a specific constant-Prandtl number line - values between $\operatorname{Pr}_{t}=0.1$ and $\operatorname{Pr}_{t}=0.5$ seem to be prevalent at $\mathrm{x} / \mathrm{D}=10.9$, and the data is much more scattered at $\mathrm{x} / \mathrm{D}=5.9$. The interpretation of the turbulent heatflux data may remain unclear until the effect of the temperature-species correlations is determined.

\section{Conclusions}

A hybrid LES / RANS simulation of sonic injection of air and helium into a Mach 1.98 flow of air has been presented. Two higher-order methods, the Piecewise Parabolic Method and the SONIC-A essentially non-oscillatory scheme have been used in the flux formulation. The upstream boundary layer structure was reasonably well predicted by the hybrid models. The hybrid LES/RANS simulations accurately predicted the center-line pressure profile upstream of the injector, but did not capture the rate of recovery of the pressure distribution downstream of the injection point. The species density contour plots agreed qualitatively with the Mie-scattering data of Gruber, et al., but some effects, such as a lateral narrowing of the plume at stations further downstream, were not captured in the calculations. The results obtained using the hybrid models indicated an improvement over the baseline Menter RANS results in almost all areas. The penetration of the jet into the cross-flow was slightly under-predicted for the helium injection case and slightly over-predicted for the air injection case. Some of the possible reasons for the discrepancies between predictions and experiment include the differences in sample size for the time averages ( 24000 images for the calculations, versus 50-100 for the experiment), inadequate resolution of the jet boundary layer as it enters the domain, and the assumption of negligible sidewall / upper wall influences in the calculations. An analysis of the LES/RANS results indicated that, for near-field mixing dominated by large-scale entrainment events, the assumption of constant Schmidt and Prandtl numbers is inadequate. As the eddies break down and the mixing occurs at smaller scales, the LES/RANS data indicated that an equilibration of mass and momentum transport rates, characterized by an approximately constant value of the Schmidt number, might occur. The results for energy / momentum transport were less certain, and further analysis involving longer spatial domains is necessary to reach any definitive conclusions.

\section{Acknowledgments}

This work is supported by NASA under Cooperative Agreement NNX07AC27A-S02 and partially by Taitech, Inc. under Contract T002-34-01. Computer time was provided by the High Performance Computing component of NCSU's Information Technologies Division (www.ncsu.edu/itd/hpc).

\section{References}

[1] Hersch, M., Povinelli, L. A., and Povinelli, F. P., “Optical Study of Sonic and Supersonic Jet Penetration from a Flat Plate into a Mach 2 Airstream,” NASA TN D-5717, March 1970.

[2] Schetz, J. A., “Interaction Shock for Transverse Injection in Supersonic Flow,” Journal of Spacecraft, Vol. 7, No. 2, Feb. 1970, pp. 143-149.

[3] Schetz, J. A., Hawkins, P. F., Lehman, H., "Structure of Highly Underexpanded Transverse Jets in a Supersonic Stream," AIAA Journal, Vol. 5, No. 5, May 1967, pp. 882-884.

[4] Zukoski, E. E., Spaid, F. W., “Secondary Injection of Gases into a Supersonic Flow,” AIAA Journal, Vol. 2, No. 10, Oct. 1964, pp. 1689-1696.

[5] Schetz, J. A. and Billig, F. S., "Penetration of Gaseous Jets into a Supersonic Stream,” AIAA Journal, Vol. 3, No. 11, Nov. 1966, pp. 1658-1665.

[6] Andrepoulos, J. and Rodi, W., "Experimental Investigations of Jets in a Crossflow,” Journal of Fluid Mechanics, Vol. 138, 1984, pp. 93-127. 
[7] McDaniel, J. C. and Graves, J., "Laser-Induced Flourescence Visualization of Transverse Gaseous Injection in a Nonreacting Supersonic Combustor,” Journal of Propulsion and Power, Vol. 4, Nov.-Dec. 1988, pp. 591-597.

[8] Hollo, S. D., McDaniel, J. C., and Hartfield Jr., R. J., “Quantitative Investigation of Compressible Mixing: Staged Transverse Injection into Mach 2 Flow,"

[9] Gruber, M. R., Nejad, A. S., and Dutton, J. C., “An Experimental Investigation of Transverse Injection from Circular and Elliptical Nozzles into a Supersonic Crossflow,” Wright Lab Technical Report WL-TR-96-2102, Jan. 1996.

[10]Gruber, M. R., Nejad, A. S., Chen, T. H., and Dutton, J. C., "Mixing and Penetration Studies of Sonic Jets in a Mach 2 Freestream,” Journal of Propulsion and Power, Vol. 11, No. 2, March-April 1995, pp. 315-323.

[11]Gruber, M. R., Nejad, A. S., Chen, T. H., and Dutton, J. C., "Compressibility Effects in Supersonic Transverse Injection Flowfields,” Physics of Fluids, Vol. 9, No. 5, May 1997, pp. 1448-1461.

[12]Gruber, M. R., Nejad, A. S., Chen, T. H., and Dutton, J. C., "Transverse Injection from Circular and Elliptical Nozzles into a Supersonic Crossflow,” Journal of Propulsion and Power, Vol. 16, No. 3, May-June 2000, pp. 449-457.

[13]Tam, C., Baurle, R. A., and Gruber, M. R., "Numerical Study of Injection into a Supersonic Crossflow," AIAA Paper 992254, June 1999.

[14]Menter, F. R., “Two-equation Eddy-viscosity Turbulence Models for Engineering Applications,” AIAA Journal, Vol. 32, No. 8, 1994, pp. 1598-1605.

[15] Wilcox, D. C., Turbulence Modeling for CFD, DCW Industries, 2nd ed., 1998.

[16] Palekar, A., Truman, C. R., and Vorobieff, P., "Prediction of Transverse Injection of a Sonic Jet in Supersonic Crossflow," AIAA Paper 2005-5366, June 2005.

[17] Maddalena, L., Campioli, T. L., and Schetz, J. A., "Experimental and Computational Investigation of Light-Gas Injectors in Mach 4.0 Crossflow,” Journal of Propulsion and Power, Vol. 22, No. 5, Sep.-Oct. 2006.

[18] Manna, P. and Chakraborty, D., "Numerical investigation of Transverse Sonic Injection in a Non-Reacting Supersonic Combustor,” Proceedings of the Institution of Mechanical Engineers, Vol. 219, Part G - Aerospace Engineering, 2005, pp. 205215.

[19] Peterson, D.M., Subbareddy, P.K, and Candler, G.V. "DES Investigations of Transverse Injection into Supersonic Crossflow using a Hybrid Unstructured Solver”, AIAA Paper 2006-0903, January, 2006.

[20] Baurle, R.A., Tam, J., Edwards, J.R., and Hassan, H.A. "Hybrid RANS/LES Approach for Cavity Flows: Blending, Algorithm, and Boundary Treatment Issues” AIAA Journal, Vol. 41, No. 8, 2003, pp. 1463-1480.

[21] Fan, T.C., Edwards, J.R., Hassan, H.A., and Baurle, R.A. "Hybrid Large-Eddy / Reynolds-Averaged Navier-Stokes Simulations of Shock-Separated Flows,” Journal of Spacecraft and Rockets, Vol. 41, No. 6, pp.897-906.

[22] Xiao, X., Edwards, J.R., Hassan, H.A., and Baurle, R.A. "Inflow Boundary Conditions for Hybrid Large-Eddy / ReynoldsAveraged Navier-Stokes Simulations” AIAA Journal, Vol. 41, No. 8, 2003, pp. 1481-1490.

[23] Edwards. J.R., Choi, J.-I., and Boles, J.A. "Hybrid LES/RANS Simulation of a Mach 5 Compression-Corner Interaction" AIAA Paper 2008-0718.

[24] Colella, P., and Woodward, P. R., “The Piecewise Parabolic Method (PPM) for Gas-Dynamical Simulations,” Journal of Computational Physics, Vol. 54, 1984, pp. 174-201.

[25] Suresh, A., and Huynh, H. T., "Numerical Experiments on a New Class of Non-Oscillatory Schemes,” AIAA Paper 920421, Jan. 1992.

[26] Walz, A. Boundary Layers of Flow and Temperature (English translation), MIT Press, 1969.

[27] Edwards, J. R., “A Low-Diffusion Flux-Splitting Scheme for Navier-Stokes Calculations,” Computers and Fluids, Vol. 26, No. 6, 1997, pp. 635-659.

[28] Gruber, M.R. and Nejad, A.S. "New Supersonic Combustion Research Facility” Journal of Propulsion and Power, Vol. 11, 1995, p.1080.

[29] Luker, J.J., Bowersoz, R.D.W., and Buter, T.A. "Influence of Curvature-Driven Favorable Pressure Gradient on Supersonic Turbulent Boundary Layer” AIAA Journal, Vol. 38, 2000, pp. 1351-1359.

[30] Choi, J.-I. and Edwards, J.R. "Compressible Boundary Layer Predictions at High Reynolds Number using Hybrid LES/RANS Methods” Abstract submitted for presentation at the $38^{\text {th }}$ AIAA Fluid Dynamics Conference, Seattle, WA.

[31] Baurle, R.A. and Eklund, D.R. “Analysis of Dual Mode Hydrocarbon Scramjet Operation at Mach 4-6.5," Journal of Propulsion and Power, Vol. 18, No. 5, 2002, pp. 990-1002

[32] Xiao, X., Hassan, H.A., Edwards, J.R., and Gaffney, R.L. "Role of Turbulent Prandtl Number on Heat Flux at Hypersonic Mach Numbers” AIAA Journal, Vol. 45, No. 4 , 2007, pp. 806-814.

[33] Xiao, X., Edwards, J.R., Hassan, H.A. and Cutler, A.D. "Variable Schmidt Number Formulation for Scramjet Applications”, AIAA Journal, Vol. 44, No. 3, 2006, pp. 593-599. 


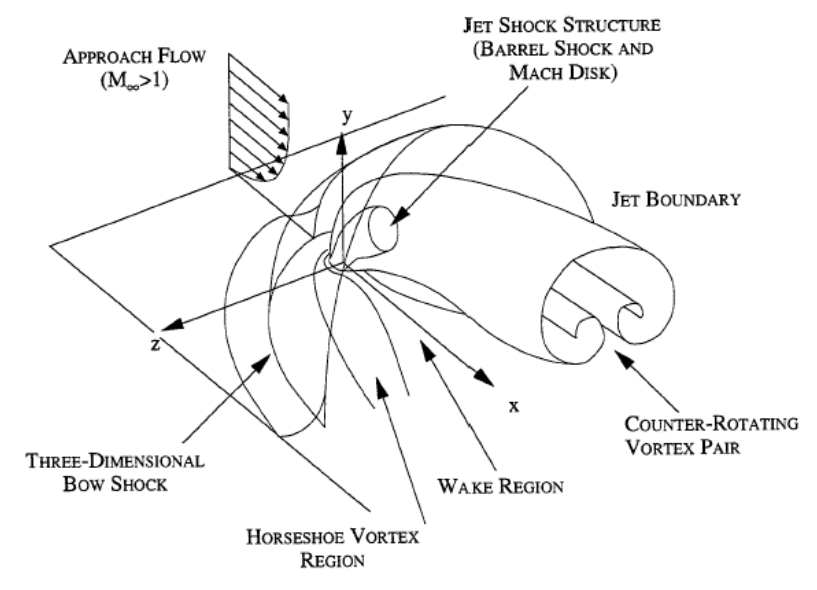

Figure 1: Perspective view of transverse injection flowfield (from Ref. 9)
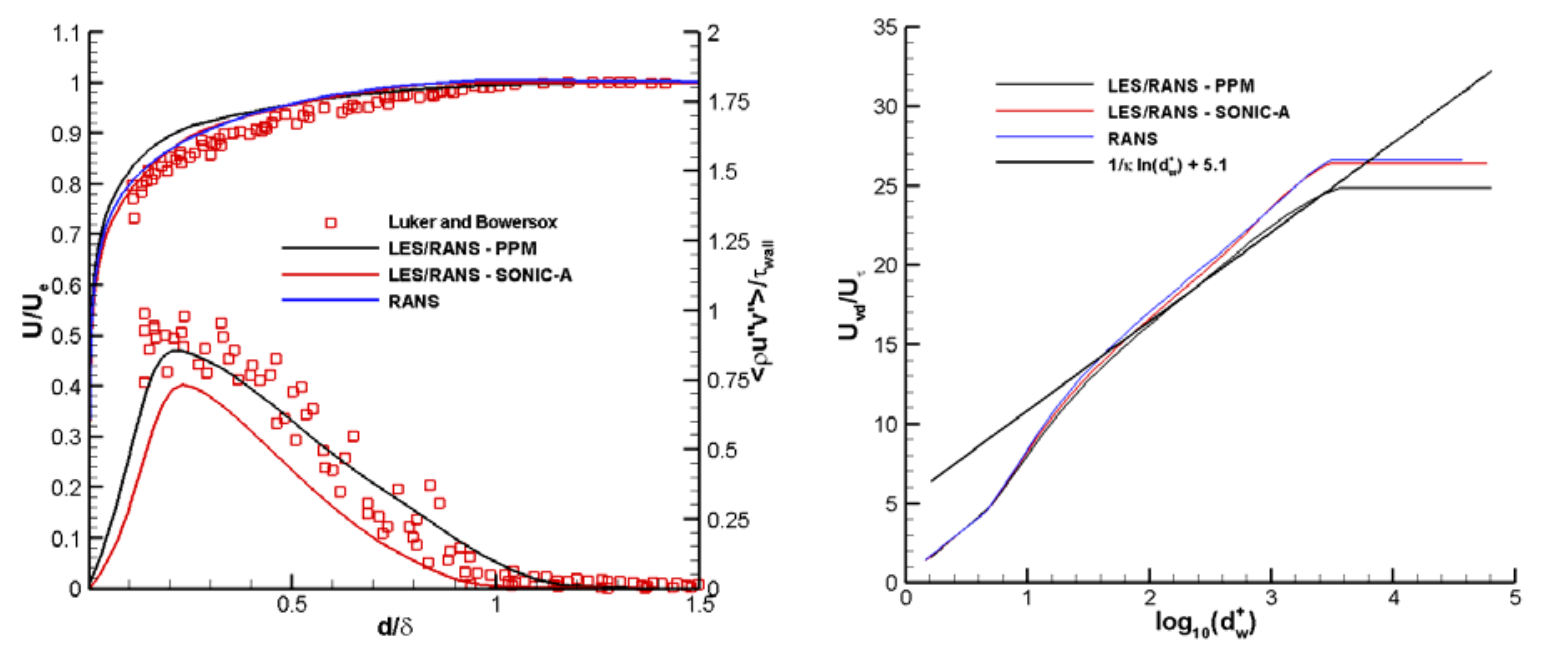

Figure 2: Velocity profiles upstream of the injector. 


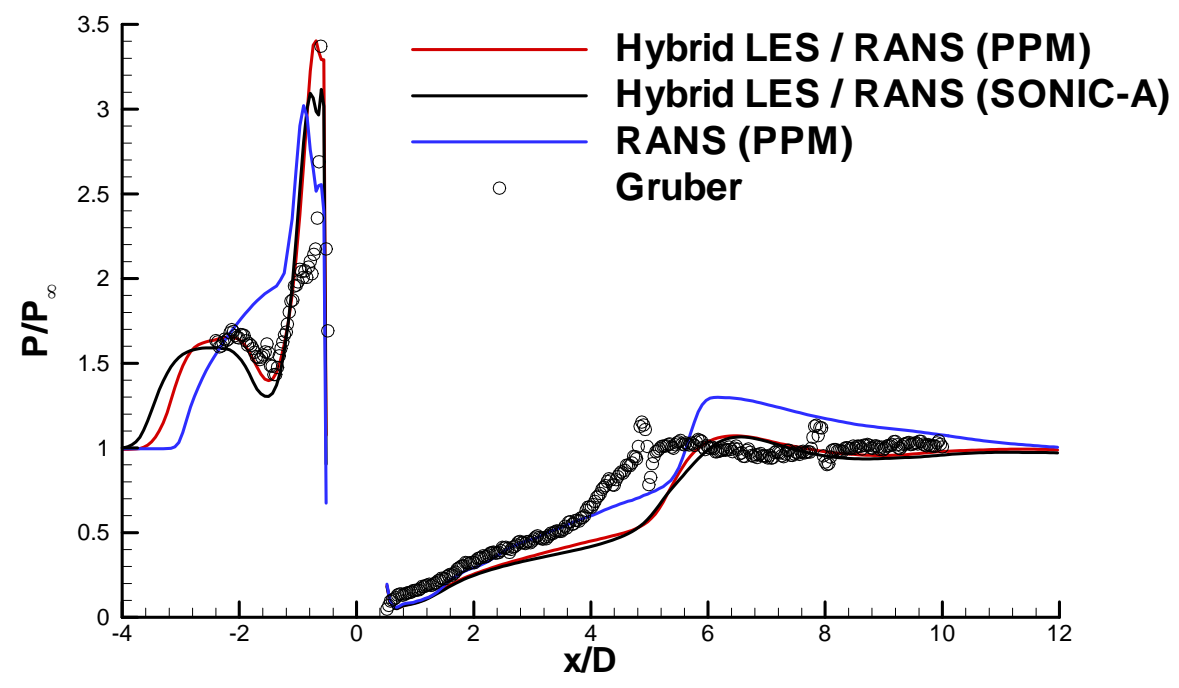

Figure 3: Center-line surface pressure profile (sonic air injection)

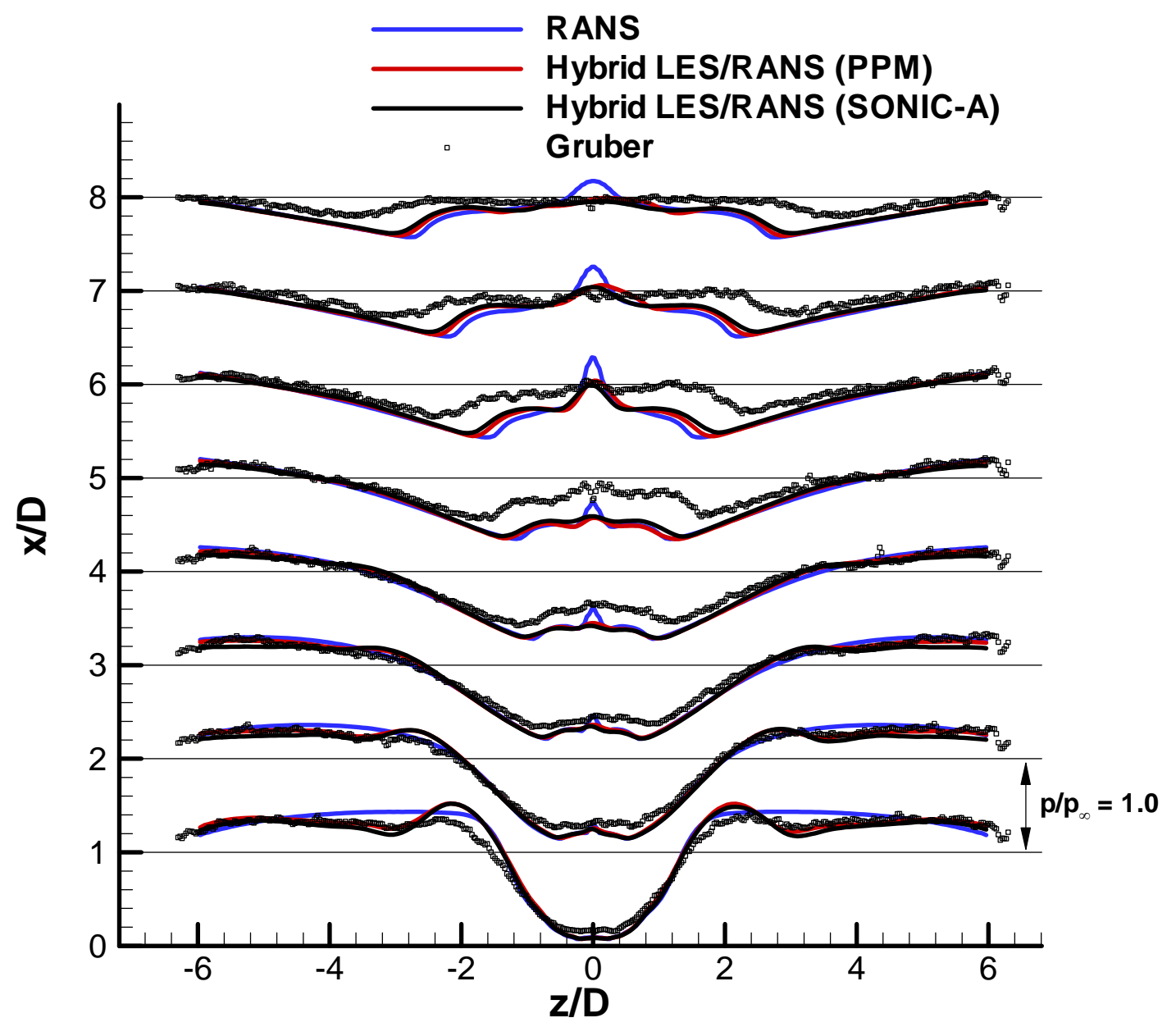

Figure 4 Transverse surface pressure profile comparisons for air injection. 

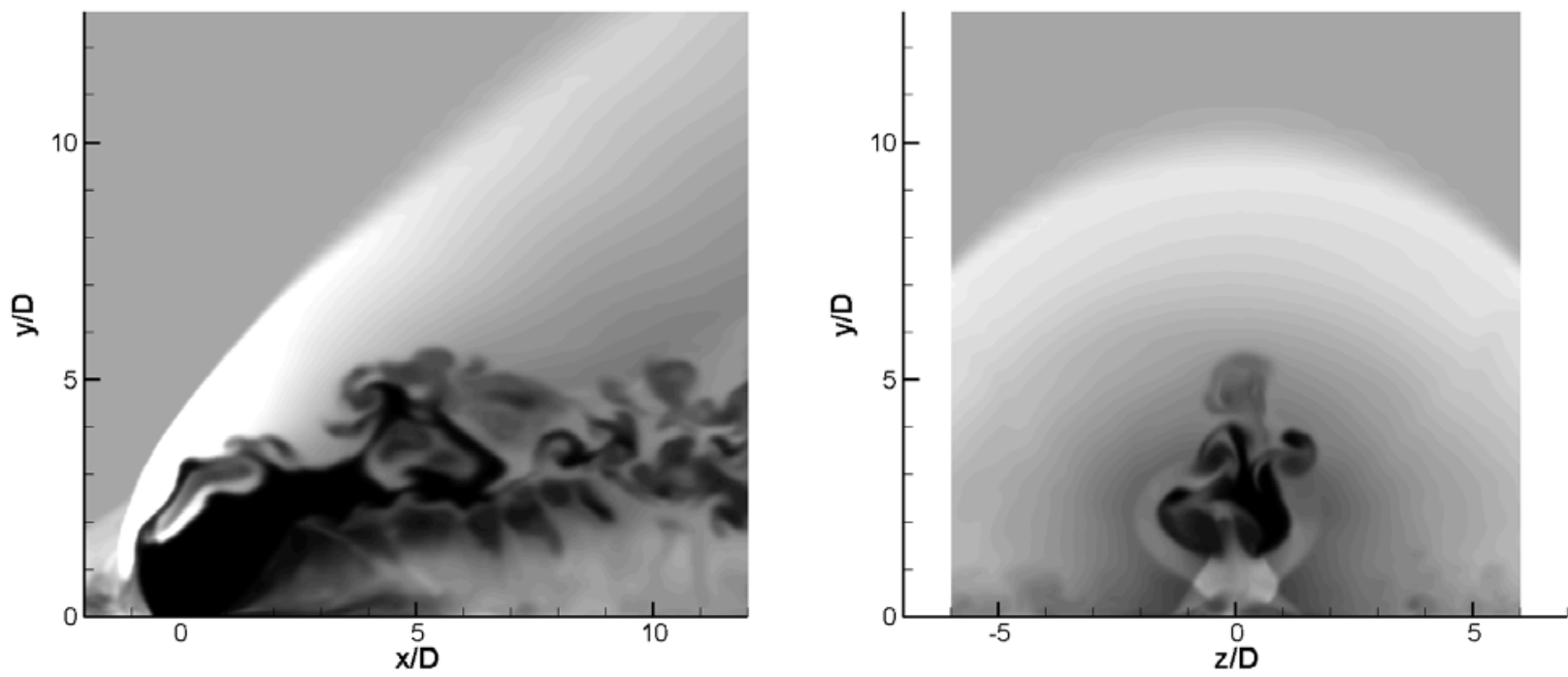

Figure 5: Species density snapshots for air injection
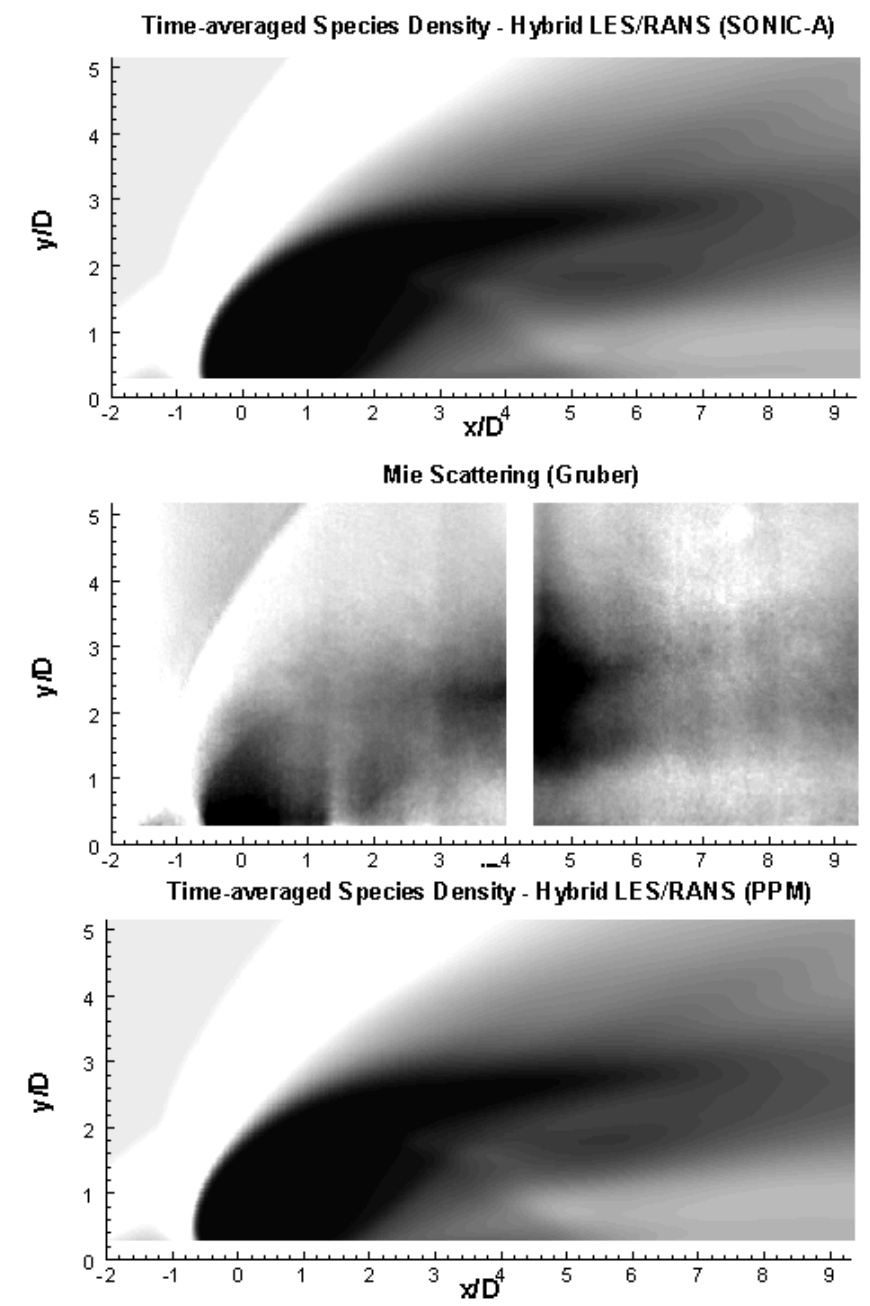

Figure 6: Center-plane Mie-scattering images and time-averaged species density contours for air injection 


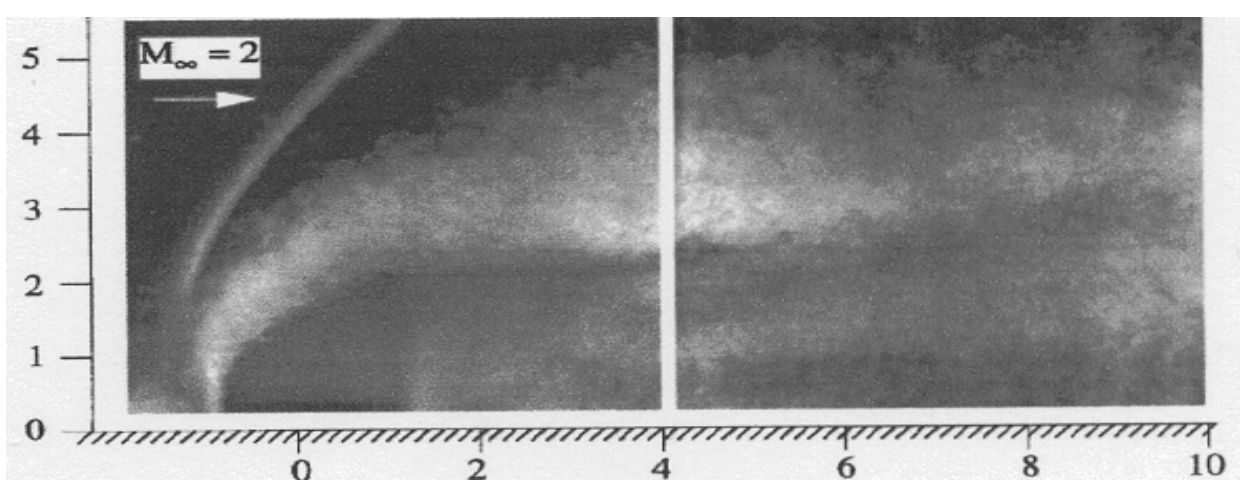

(a) Intensity standard deviation (Ref. 11)

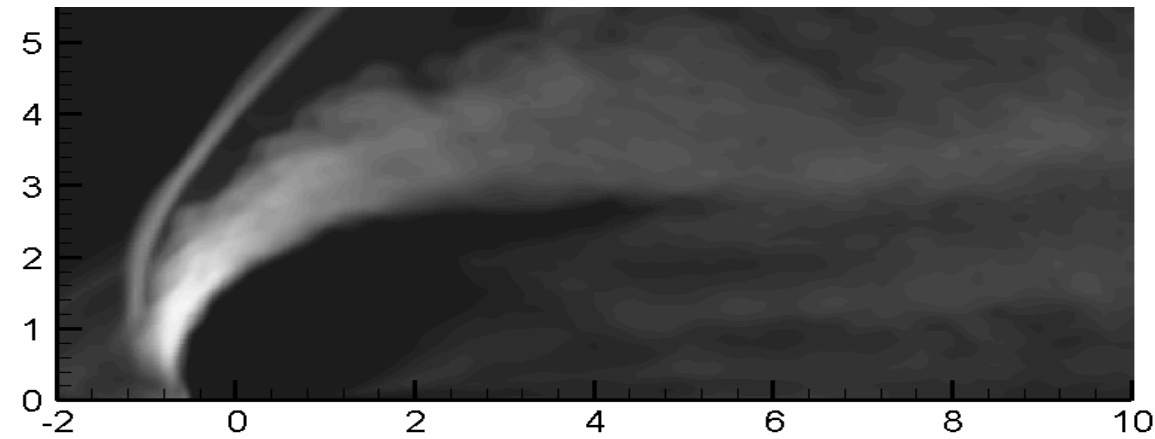

(b) Standard deviation of inlet air density

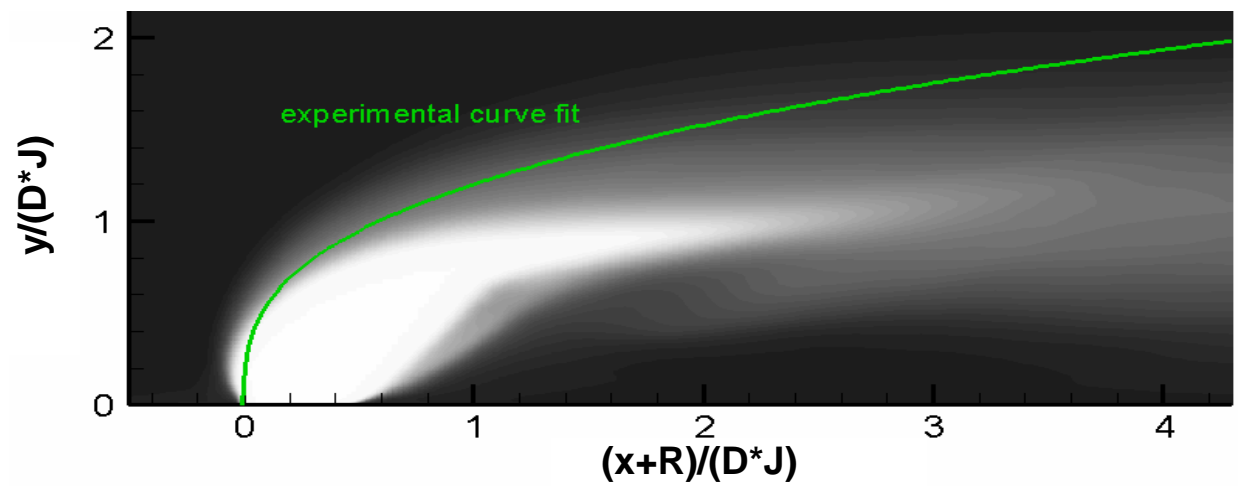

(c) Time-averaged mass fraction

Figure 7: Center-plane time-averaged mass fraction and density standard deviation comparison for sonic air injection 


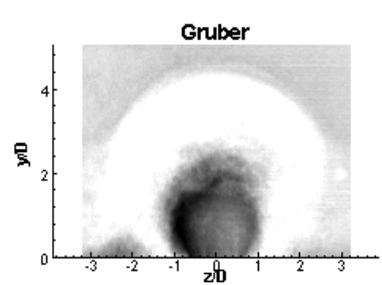

$x / D=0$
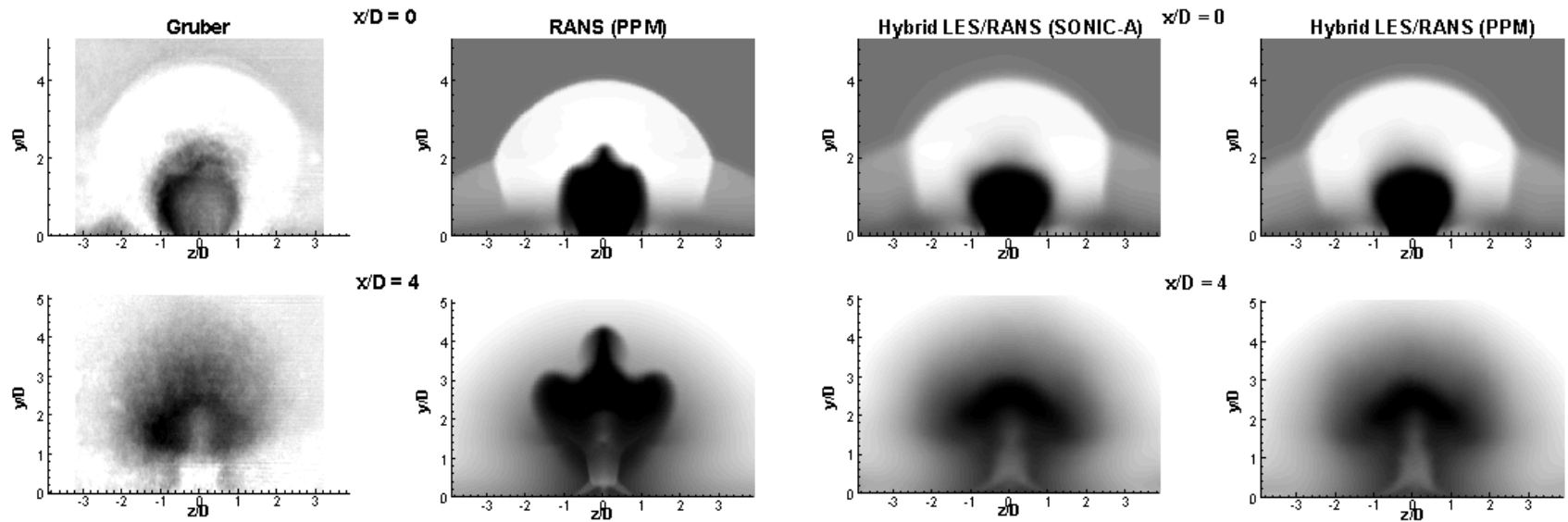

$\mathrm{x} / \mathrm{D}=\mathbf{4}$
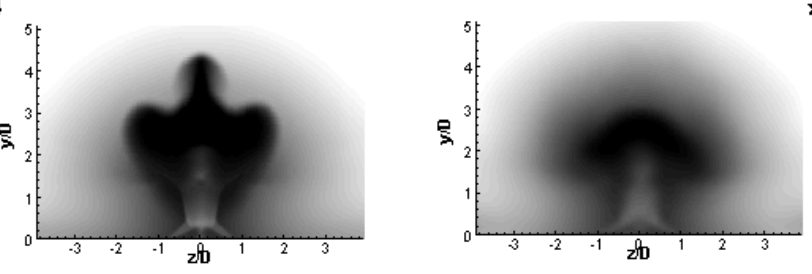

$\mathrm{x} / \mathrm{D}=4$
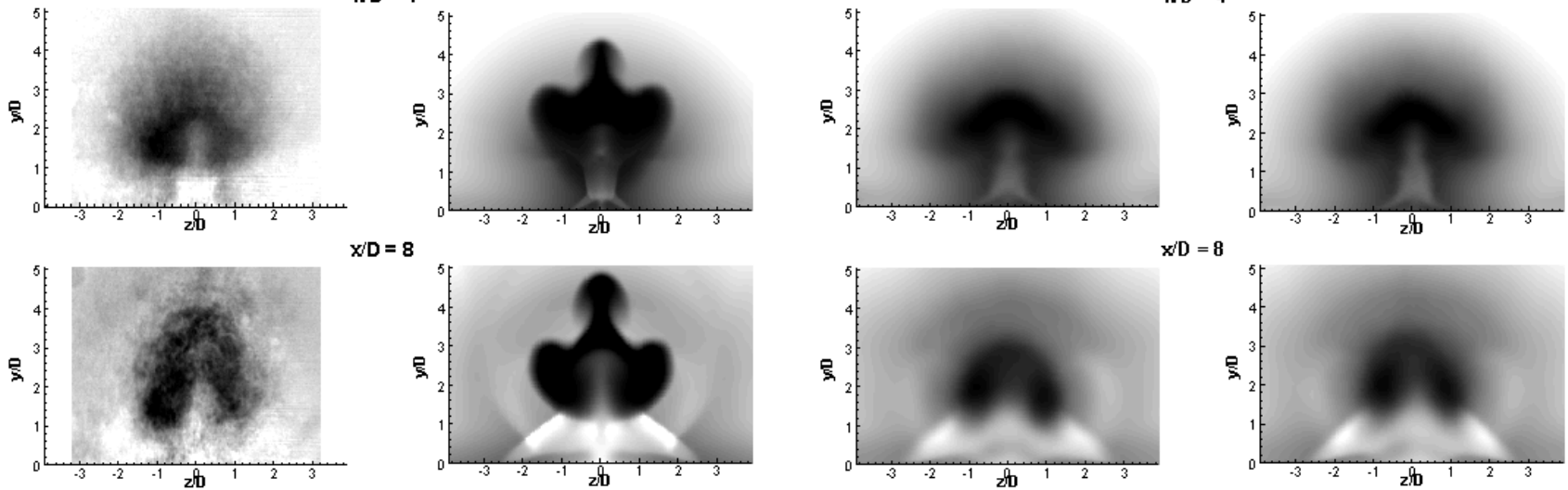

$x / D=8$
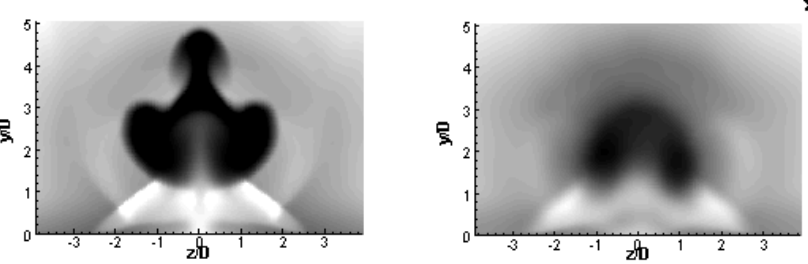

$\mathrm{x} / \mathrm{D}=\mathbf{8}$
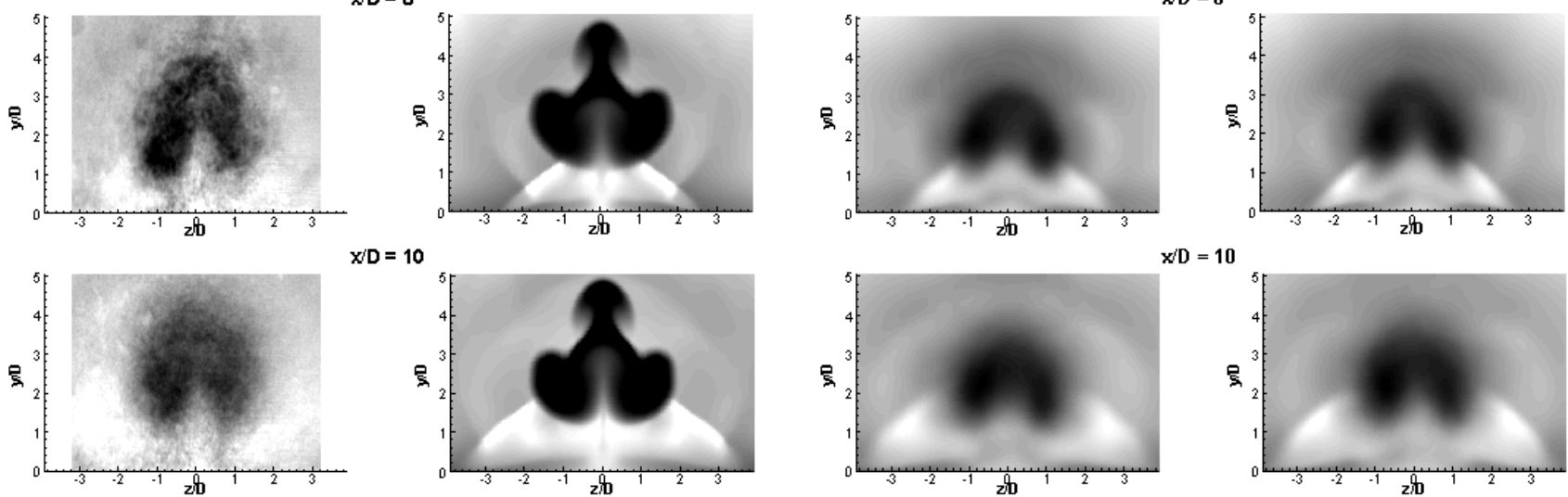

$x / D=10$
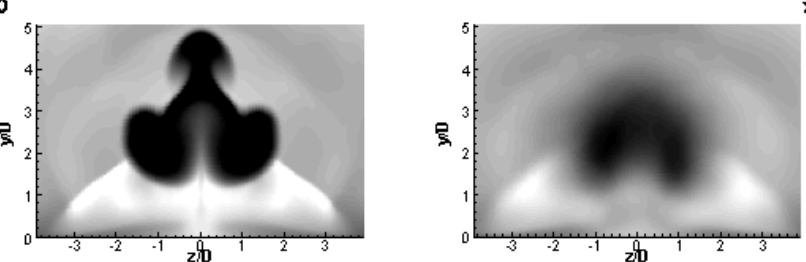

$x / D=10$

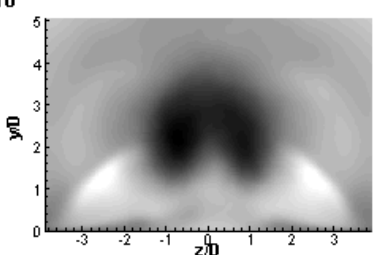

Figure 8: Transverse experimental Mie-scattering images and computational contours of species density for sonic injection of air 

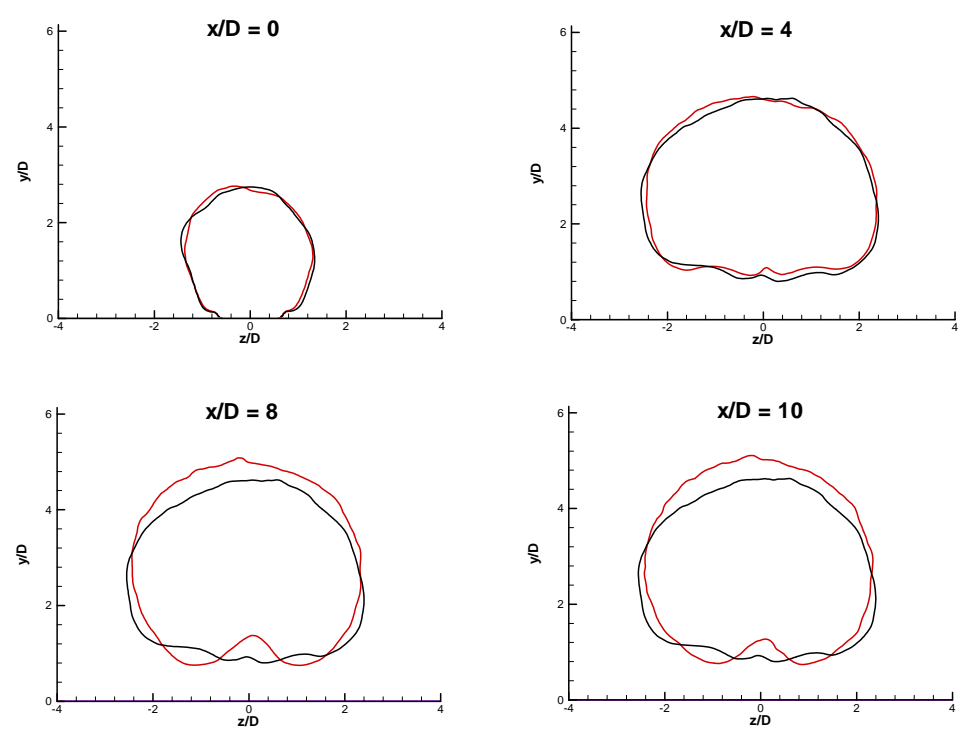

Figure 9: Penetration contours for air injection - PPM (red) versus SONIC-A (black)
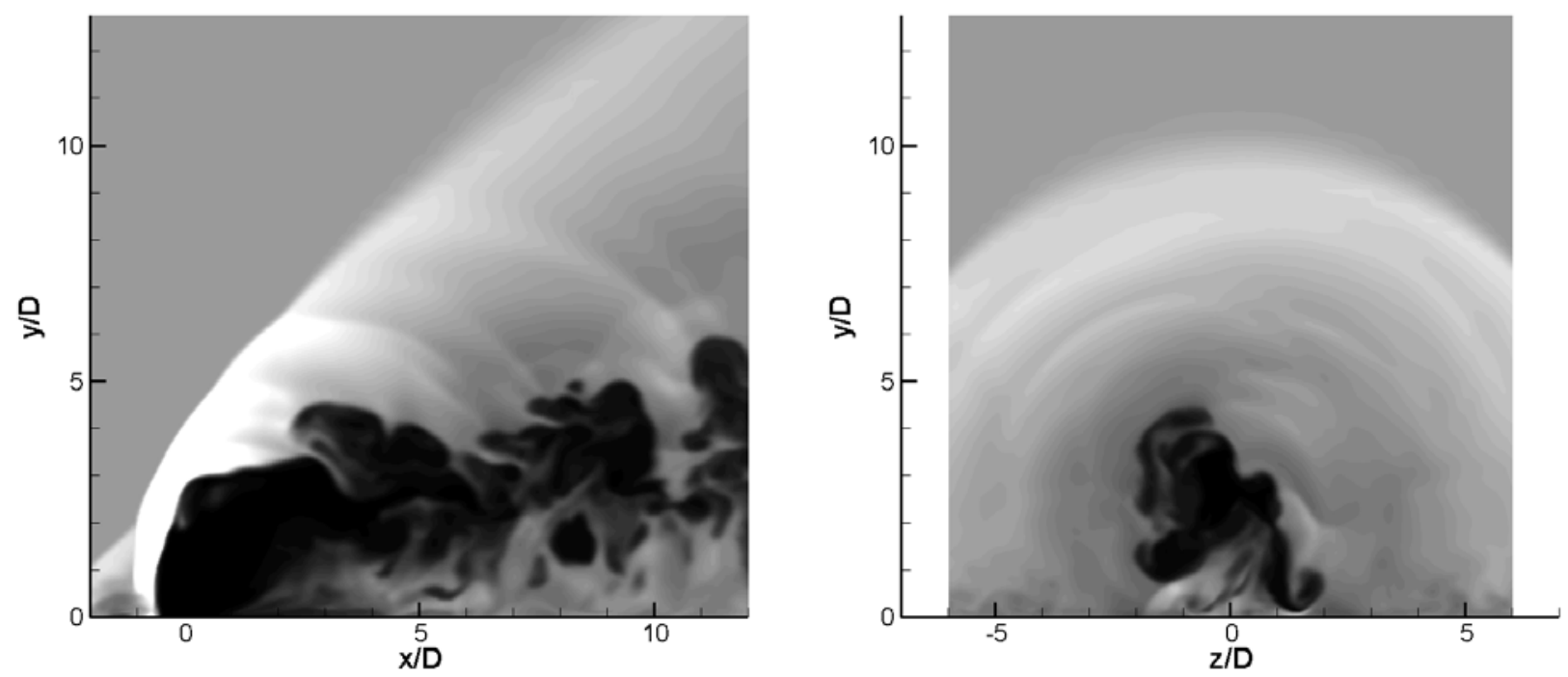

Figure 10: Species density snapshots for helium injection. 


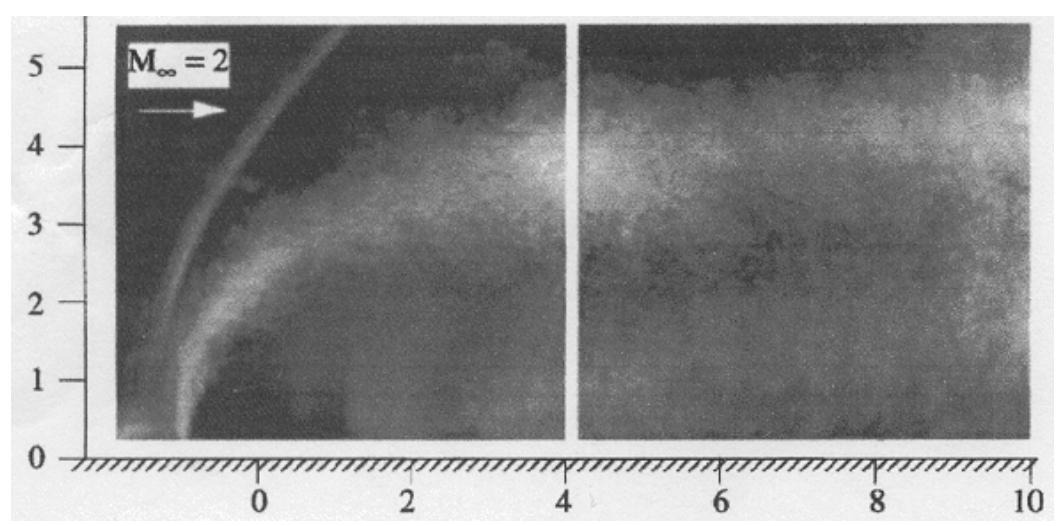

(a) Intensity standard deviation (Ref. 11)

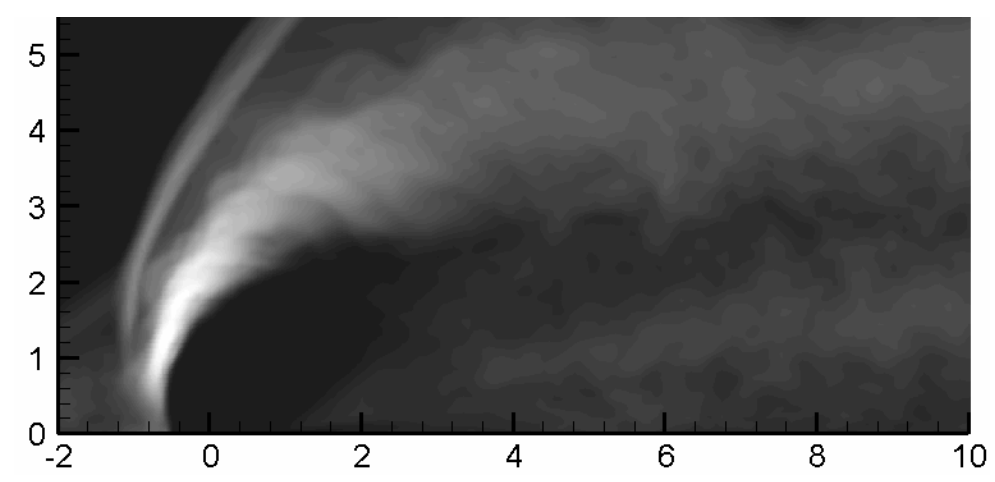

(b) Standard deviation of inlet helium density

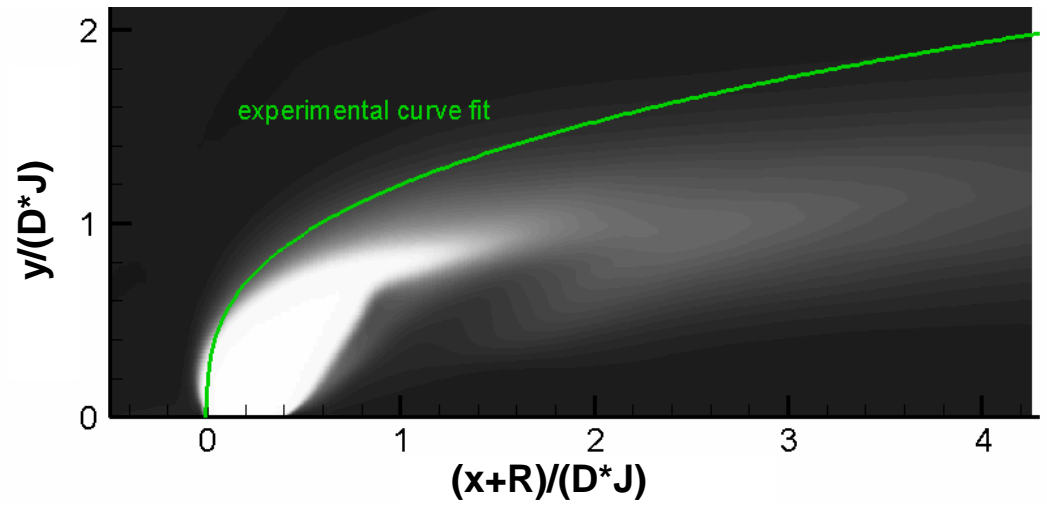

(c) Time-averaged mass fraction

Figure 11: Center-plane time-averaged data comparison for sonic helium injection. 

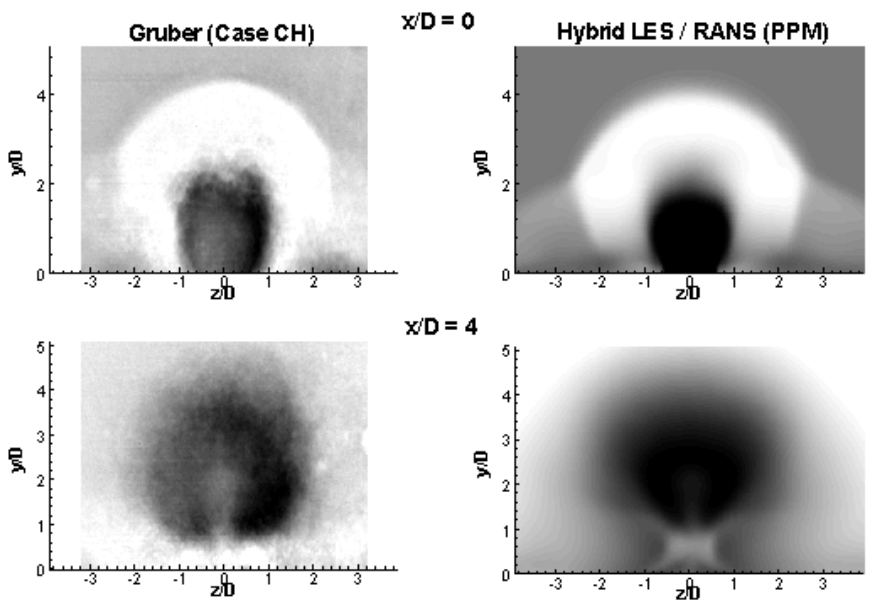

$\mathrm{x} / \mathrm{D}=\mathbf{4}$
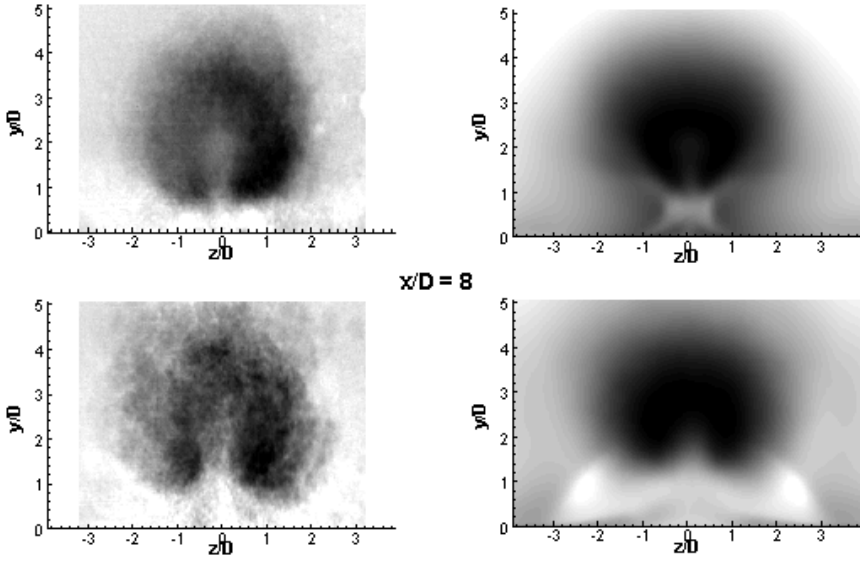

$\mathrm{x} / \mathrm{D}=8$

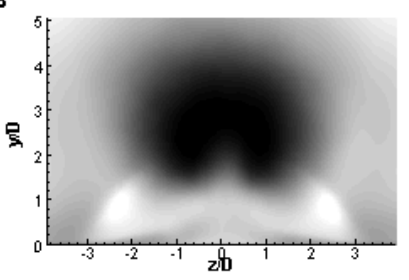

$x / D=10$
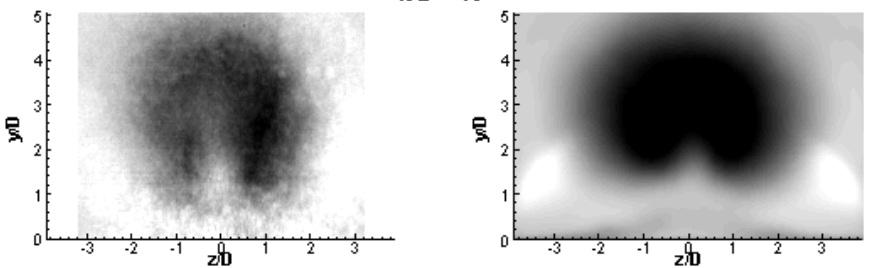

Figure 12: Transverse experimental Mie-scattering images and computational contours of species density of the bulk-flow of air with sonic injection of helium.
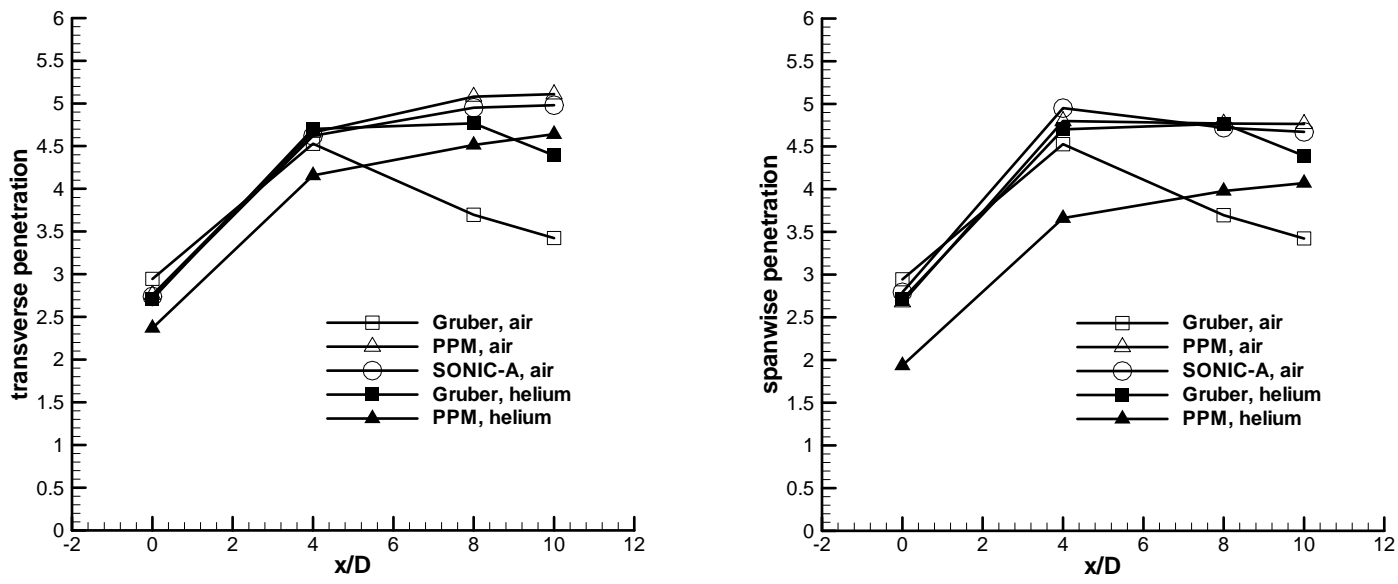

Figure 13 Penetration profiles - transverse (left) and spanwise (right) . 


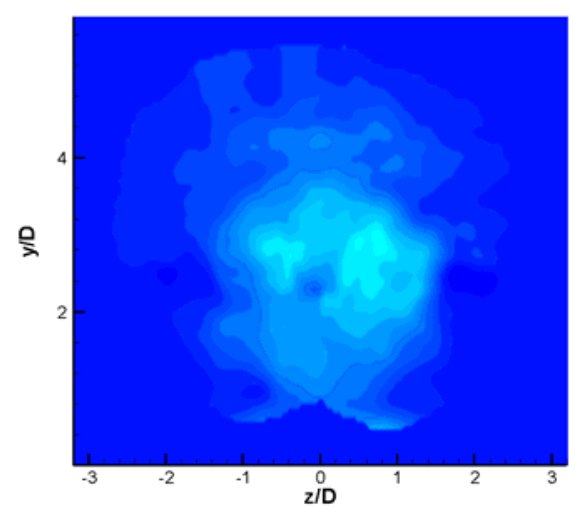

a.) momentum

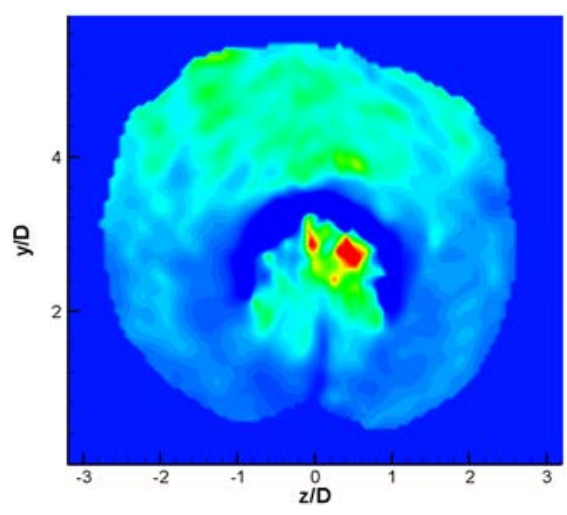

b.) mass

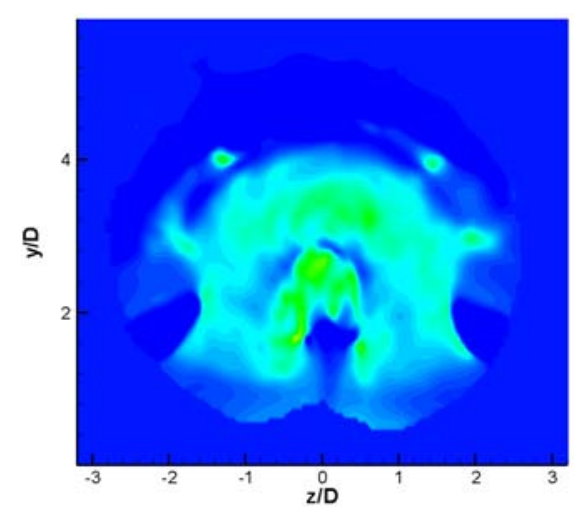

c.) energy

Figure 14: Contours of effective eddy viscosities for momentum, mass, and energy transfer $(x / D=10.9$; air injection; scale ranges from 0 (blue) to 0.5 (red) $\left(\mathrm{m}^{2} / \mathrm{s}\right)$ )

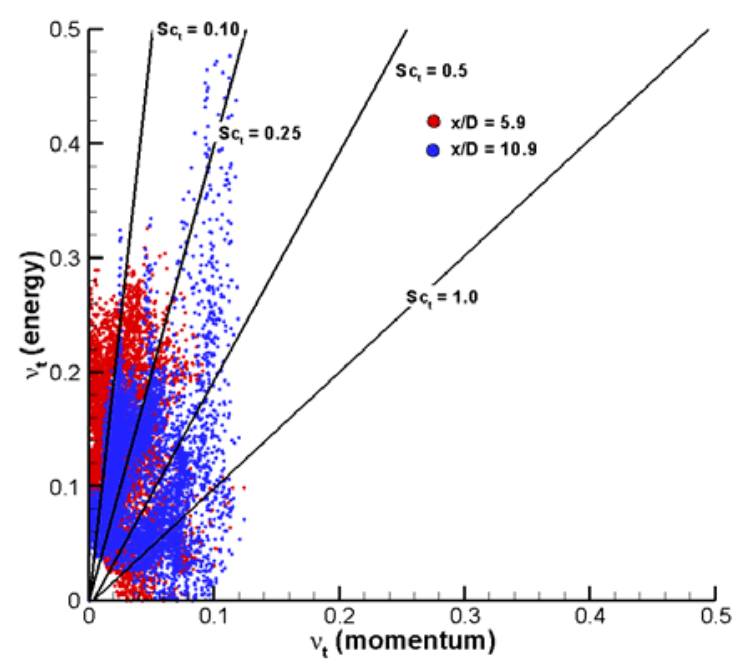

a.) momentum vs mass

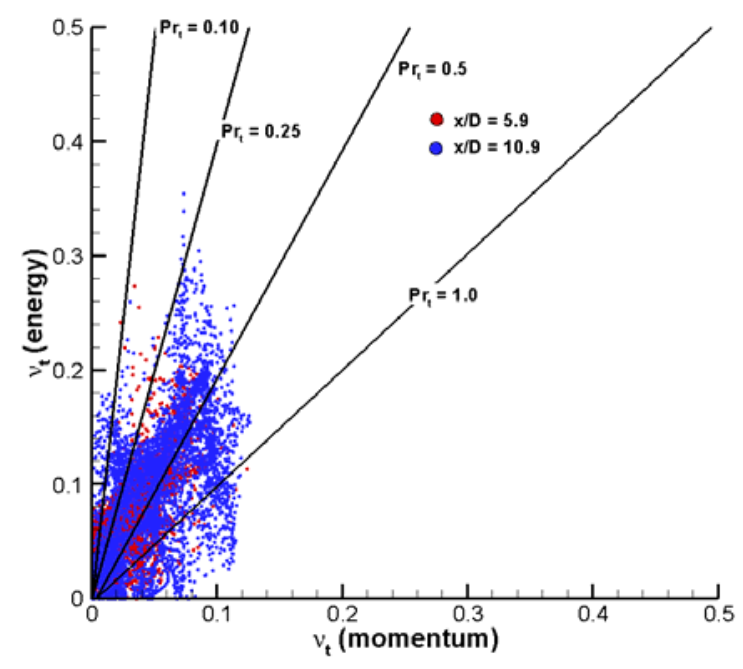

b.) momentum vs energy

Figure 15: Scatter plots of momentum eddy viscosity versus mass and energy eddy viscosities (air injection) 


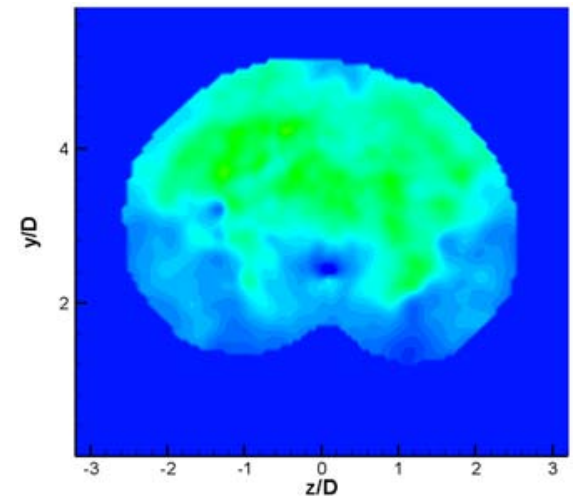

a.) momentum

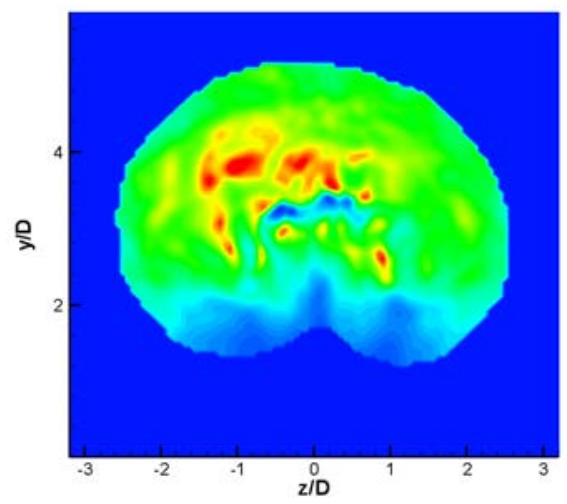

b.) mass

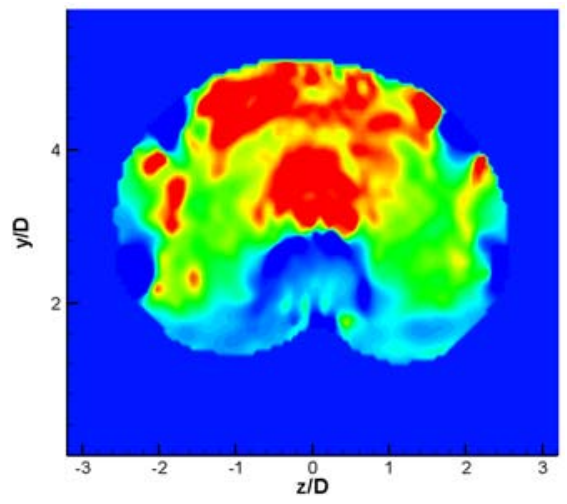

c.) energy

Figure 16: Contours of effective eddy viscosities for momentum, mass, and energy transfer $(x / D=10.9$; helium injection; scale ranges from 0 (blue) to 0.5 (red) $\left(\mathrm{m}^{2} / \mathrm{s}\right)$ )

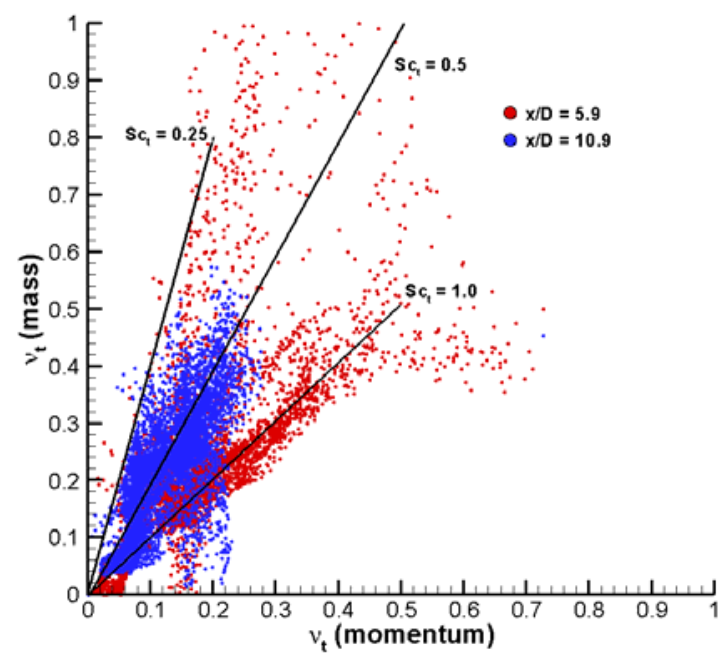

a.) momentum vs mass

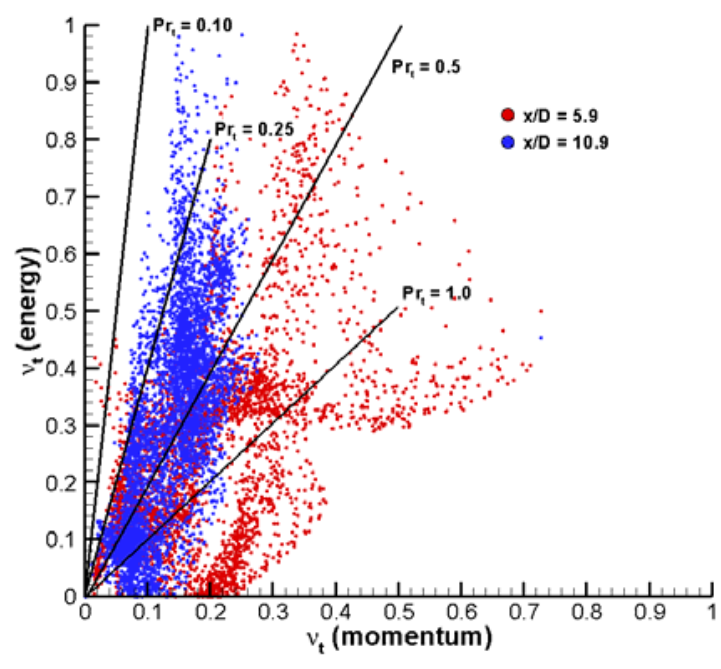

b.) momentum vs energy

Figure 17: Scatter plots of momentum eddy viscosity versus mass and energy eddy viscosities (helium injection) 